\title{
Exact Radiation and Scattering for an Elliptic Metal Cylinder at the Interface between Isorefractive Half-spaces
}

\author{
Danilo Erricolo, Senior Member, IEEE, Piergiorgio L. E. Uslenghi, Fellow, IEEE
}

\begin{abstract}
An elliptic metal cylinder located at the interface between two isorefractive half-spaces, with its cross-sectional major axis either parallel or perpendicular to the interface is considered. The problems of scattering of an incident plane wave with arbitrary polarization and direction of incidence, and of radiation from an electric or magnetic line source parallel to the cylinder axis are solved exactly. The particular case of a metal strip either parallel or perpendicular to the interface is examined in detail. Several numerical results for far fields and surface currents are presented.
\end{abstract}

Index Terms-Electromagnetic radiation, electromagnetic scattering, complex media.

\section{INTRODUCTION}

$\mathbf{N}$ EW materials with interesting electromagnetic and electronic properties have recently been introduced for a variety of applications: materials with various types of anisotropy for substrates, radomes, RAM, FSS such as ferrites, ceramics, fiber composites, honeycomb structures; bianisotropic materials; chiral materials; metamaterials; etc. Sophisticated computer codes have been developed, both in frequency and time domains, to analyze the electromagnetic behavior of complex structures containing such penetrable materials. The accuracy of such codes may be tested by comparison with other codes, with measurements data, or with exact solutions of boundaryvalue problems for structures of sufficient complexity to provide challenging and meaningful comparisons. Until a few years ago, the only exact solutions known for penetrable bodies involved the circular cylinder and the sphere. The recent introduction of isorefractive materials, i.e. materials having the same refractive index but different intrinsic impedances, has led to the development of several new exact solutions, usually by the method of separation of variables, that provide realistic tests for computer codes while enriching the catalog of available canonical solutions.

Several problems involving two-dimensional isorefractive bodies have been solved exactly [1], with particular attention to the isorefractive wedge both in the frequency domain [2], [3], [4] and in the time domain [5]. Exact solutions have also been obtained for three-dimensional isorefractive bodies

The authors are with the Department of Electrical and Computer Engineering, University of Illinois at Chicago, 851 South Morgan St., Chicago, Illinois 60607, USA. E-mail: derricol@ece.uic.edu, uslenghi@uic.edu. This research was supported by the U.S. Department of Defense under MURI grant F49620-01-1-0436. Additionally, this work was supported in part by a grant of computer time from the DOD High Performance Computing Modernization Program at ASC. of revolution [6], [7] and for cavities filled with isorefractive material in two dimensions [8], [9] and in three dimensions [10]. Some of these results have been compared successfully with numerical solutions of integral equations formulations of the same problems [11], [12], [13].

In this work, the two-dimensional problem of a PEC cylinder of elliptical cross section located at the plane interface between isorefractive half-spaces is considered. The major axis of the cross-sectional ellipse is either in the plane of the interface (hereinafter called "parallel configuration", see Fig. 1 ), or perpendicular to the interface (hereinafter called "perpendicular configuration", see Fig. 2). The problem is solved exactly for a primary plane wave with arbitrary polarization and direction of incidence in Section III, and for a primary electric or magnetic line source parallel to the cylinder axis in Section IV. The particular case in which the elliptic cylinder is flattened onto a metal strip is discussed in Section $\mathrm{V}$, and some numerical results for the far fields (RCS) and surface currents on the cylinder are shown and discussed in Section VI. The analysis is conducted in the frequency domain, with time-dependence factor $\exp (+j \omega t)$ omitted throughout.

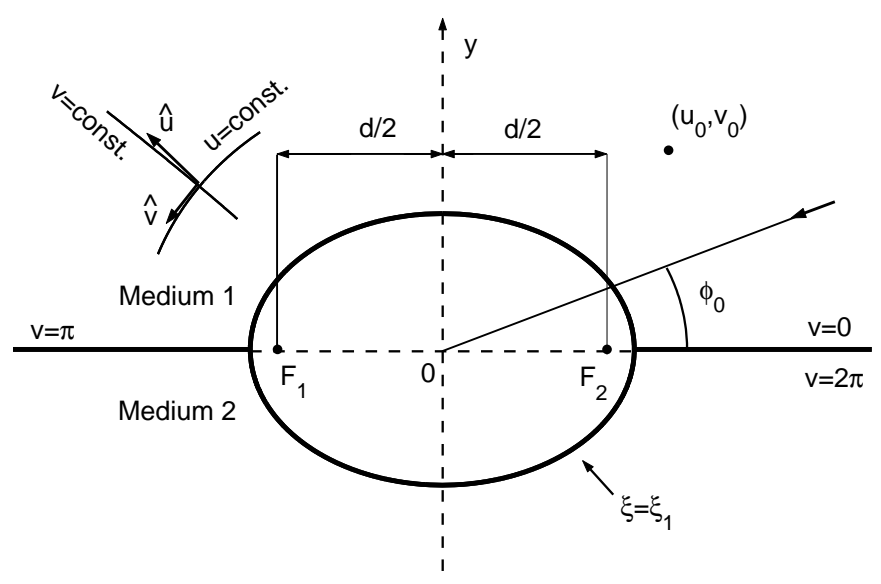

Fig. 1. Geometry of the problem for parallel configuration.

\section{STATEMENT OF THE PROBLEM}

Two configurations are analyzed. In the parallel configuration, whose cross section is shown in Fig. 1, the planar interface $y=0$ separates two half-spaces. The region $y>0$ is occupied by medium 1 , characterized by permittivity $\varepsilon_{1}$ and permeability $\mu_{1}$, whereas the region $y<0$ is occupied by 


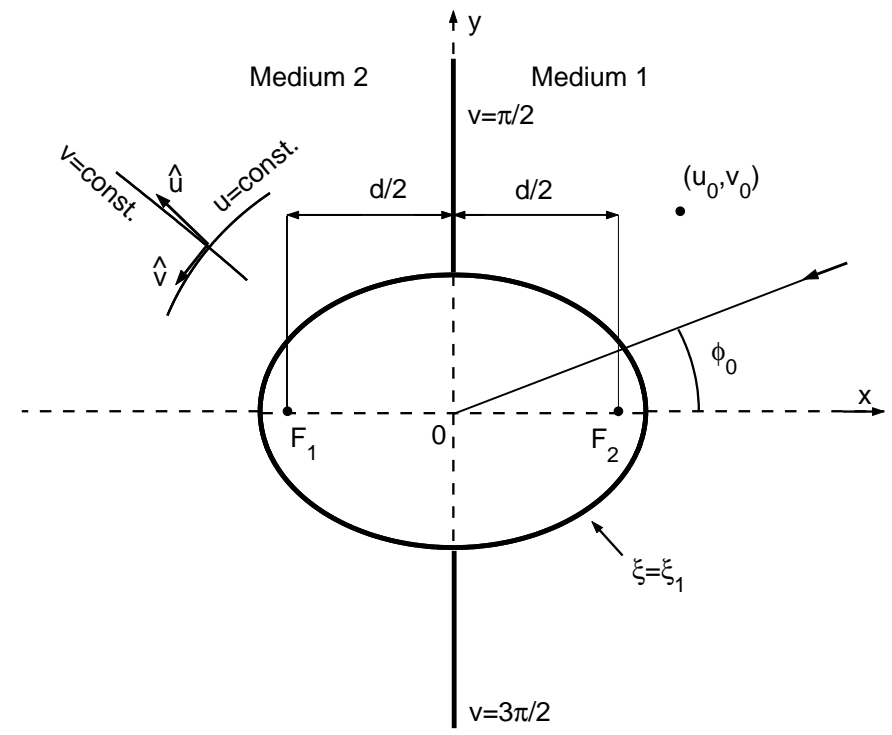

Fig. 2. Geometry of the problem for perpendicular configuration.

medium 2, characterized by permittivity $\varepsilon_{2}$ and permeability $\mu_{2}$. The two media are isorefractive, i.e.

$$
\varepsilon_{1} \mu_{1}=\varepsilon_{2} \mu_{2}
$$

so that the propagation constant $k$ and the wavelength $\lambda$ are the same in both media:

$$
k=2 \pi / \lambda=\omega \sqrt{\varepsilon_{h} \mu_{h}},(h=1,2)
$$

whereas the intrinsic impedances

$$
Z_{h}=\sqrt{\mu_{h} / \varepsilon_{h}},(h=1,2)
$$

are, in general, different from each other.

A metallic elliptic cylinder straddles the interface between the media. Its cross section in any plane $z=$ constant is an ellipse with interfocal distance $F_{1} F_{2}=d$ and foci $F_{1}$ and $F_{2}$ that lie in the plane $y=0$ of the interface. The rectangular coordinates $(x, y, z)$ are related to the elliptic coordinates $(u, v, z)$ by:

$$
\begin{aligned}
& x=\frac{d}{2} \cosh u \cos v, \\
& y=\frac{d}{2} \sinh u \sin v, \\
& z=z
\end{aligned}
$$

where $0 \leq u<\infty, 0 \leq v \leq 2 \pi,-\infty<z<\infty$. It is sometimes useful to introduce the coordinates

$$
\xi=\cosh u, \eta=\cos v
$$

with $1 \leq \xi<\infty$ and $-1 \leq \eta \leq 1$. The inverse transformation from cartesian coordinates $(x, y)$ to elliptic coordinates $(\xi, \eta)$ is reported here for convenience:

$$
\begin{aligned}
& \xi= \\
& \sqrt{\frac{4\left(x^{2}+y^{2}\right)+d^{2}+\sqrt{16\left(x^{2}+y^{2}\right)^{2}+d^{4}-8 d^{2}\left(x^{2}-y^{2}\right)}}{2 d^{2}}}
\end{aligned}
$$

and

$$
\eta=\frac{2 x}{d \xi}
$$

The surface of the PEC elliptic cylinder is the coordinate surface $\xi=\xi_{1}$, whereas the interface $y=0$ corresponds to $v=0,2 \pi$ for $x>0$ and to $v=\pi$ for $x<0$.

In the perpendicular configuration, whose cross section is shown in Fig. 2, the interface between medium $1(x>0)$ and medium $2(x<0)$ is the plane $x=0$; thus, the interface corresponds to $v=\pi / 2$ for $y>0$ and to $v=3 \pi / 2$ for $y<0$. The interfocal segment $F_{1} F_{2}$ is now perpendicular to the interface.

For either configuration, it is expedient to introduce the parameter

$$
c=\frac{k d}{2} .
$$

as well as the reflection coefficient $R$ and transmission coefficient $T$ for the electric field when incidence on the interface occurs from medium 1 :

$$
R=\frac{1-\zeta}{1+\zeta}, \quad T=\frac{2}{1+\zeta},
$$

where

$$
\zeta=Z_{1} / Z_{2}
$$

note that

$$
1+R=T, \quad 1-R=\zeta T .
$$

The primary field is either a plane wave or a line source. For a plane wave incident from the first quadrant and propagating in a direction that forms the angle $\varphi_{0}$ with the negative $\mathrm{x}$-axis and the angle $\left(\pi / 2-\varphi_{0}\right)$ with the negative y-axis (see Figs. 1 and 2), either the electric or the magnetic field is taken as parallel to the $\mathrm{z}$-axis and given by the phasor

$$
\begin{aligned}
& e^{j k\left(x \cos \varphi_{0}+y \sin \varphi_{0}\right)}= \\
= & \sqrt{8 \pi} \sum_{m=0}^{\infty} j^{m}\left[\frac{1}{N_{m}^{(e)}} \operatorname{Re}_{m}^{(1)}(c, \xi) \operatorname{Se}_{m}(c, \eta) \operatorname{Se}_{m}\left(c, \cos \varphi_{0}\right)\right. \\
+ & \left.\frac{1}{N_{m}^{(o)}} \operatorname{Ro}_{m}^{(1)}(c, \xi) \mathrm{So}_{m}(c, \eta) \operatorname{So}_{m}\left(c, \cos \varphi_{0}\right)\right],
\end{aligned}
$$

where Re, $o_{m}^{(1)}$ are the even and odd radial Mathieu functions of the first kind, Se, $\mathrm{o}_{m}$ are the even and odd angular Mathieu functions, and $N_{m}^{(e),(o)}$ are normalization coefficients; here and in the following, the Stratton-Chu notation is adopted [14], [15].

For an isotropic electric or magnetic line source parallel to the z-axis and located in the first quadrant at $\left(x_{0}, y_{0}\right) \equiv$ $\left(u_{0}, v_{0}\right) \equiv\left(\xi_{0}, \eta_{0}\right)$, the primary electric or magnetic field is given by the Hankel function of zero order and of the second kind:

$$
\begin{aligned}
& H_{0}^{(2)}(k r)= \\
& 4 \sum_{m=0}^{\infty}\left[\frac{1}{N_{m}^{(e)}} \operatorname{Re}_{m}^{(1)}\left(c, \xi_{<}\right) \operatorname{Re}_{m}^{(4)}\left(c, \xi_{>}\right) \operatorname{Se}_{m}\left(c, \eta_{0}\right) \operatorname{Se}_{m}(c, \eta)+\right. \\
& \left.\frac{1}{N_{m}^{(o)}} \operatorname{Ro}_{m}^{(1)}\left(c, \xi_{<}\right) \operatorname{Ro}_{m}^{(4)}\left(c, \xi_{>}\right) \operatorname{So}_{m}\left(c, \eta_{0}\right) \operatorname{So}_{m}(c, \eta)\right]
\end{aligned}
$$


where $\xi_{<}\left(\xi_{>}\right)$is the smaller (larger) between $\xi$ and $\xi_{0}, \operatorname{Re}, o_{m}^{(4)}$ are the even and odd radial Mathieu functions of the fourth kind, and $r$ is the distance of the observation point from the line source:

$$
r=\sqrt{\left(x-x_{0}\right)^{2}+\left(y-y_{0}\right)^{2}} .
$$

The solutions listed in the following sections are obtained by imposing the boundary conditions at the planar interface (i.e., continuity of the tangential electric and magnetic fields across the interface), zero tangential electric field at the surface of the PEC cylinder, and the two-dimensional radiation condition at infinity for all fields generated by primary and secondary sources of finite extent. No details are provided, but the results can be verified by using the properties of Mathieu functions listed in the appendix of [8]. For the case of perpendicular configuration, those properties must be supplemented by the following:

$$
\begin{aligned}
\left.\operatorname{Se}_{2 \ell+1}(c, \eta)\right|_{v=\pi / 2,3 \pi / 2} & =0, \\
\left.\operatorname{So}_{2 \ell}(c, \eta)\right|_{v=\pi / 2,3 \pi / 2} & =0, \\
\left.\frac{\partial}{\partial v} \operatorname{Se}_{2 \ell}(c, \eta)\right|_{v=\pi / 2,3 \pi / 2} & =0, \\
\left.\frac{\partial}{\partial v} \operatorname{So}_{2 \ell+1}(c, \eta)\right|_{v=\pi / 2,3 \pi / 2} & =0,
\end{aligned}
$$

where $\ell=0,1,2, \ldots$ is any non-negative integer.

\section{Plane WaVe incidence}

The most general polarization of the incident plane wave is easily obtained by superposition of the solutions for Epolarization (electric field parallel to the $\mathrm{z}$-axis) and $\mathrm{H}$ polarization (magnetic field parallel to the z-axis).

\section{A. Parallel configuration with E-polarization.}

If the incident plane wave has an electric field $\underline{E}^{i}=\hat{z} E_{z}^{i}$ parallel to the z-axis with $E_{z}^{i}$ given by (12), then the total electric fields $E_{1 z}$ in medium 1 and $E_{2 z}$ in medium 2 may be written as

$$
\begin{aligned}
& E_{1 z}=E_{z}^{i}+E_{z}^{r}+E_{1 z}^{s}, \\
& E_{2 z}=E_{z}^{t}+E_{2 z}^{s},
\end{aligned}
$$

where $E_{z}^{r}$ and $E_{z}^{t}$ are the reflected and transmitted fields that would occur in the absence of the metallic cylinder, whereas $E_{h z}^{s}(h=1,2)$ are the scattered fields due to the presence of the cylinder. The fields $E_{z}^{r}$ and $E_{z}^{t}$ are associated with reflected and transmitted plane waves, whereas $E_{h z}^{s}$ must satisfy the radiation condition.

It is found that:

$$
\begin{aligned}
& E_{z}^{i}+E_{z}^{r}= \\
& =\sqrt{8 \pi} \sum_{m=0}^{\infty} j^{m}\left[\frac{1+R}{N_{m}^{(e)}} \operatorname{Re}_{m}^{(1)}(c, \xi) \operatorname{Se}_{m}(c, \eta) \operatorname{Se}_{m}\left(c, \cos \varphi_{0}\right)\right. \\
& \left.+\frac{1-R}{N_{m}^{(o)}} \operatorname{Ro}_{m}^{(1)}(c, \xi) \operatorname{So}_{m}(c, \eta) \operatorname{So}_{m}\left(c, \cos \varphi_{0}\right)\right]
\end{aligned}
$$

$$
\begin{aligned}
& E_{z}^{t}=T E_{z}^{i} \\
& E_{h z}^{s}= \\
& \sqrt{8 \pi} \sum_{m=0}^{\infty} j^{m}\left[\frac{a_{h, m}^{(e)}}{N_{m}^{(e)}} \operatorname{Re}_{m}^{(4)}(c, \xi) \operatorname{Se}_{m}(c, \eta) \operatorname{Se}_{m}\left(c, \cos \varphi_{0}\right)\right. \\
& \left.+\frac{a_{h, m}^{(o)}}{N_{m}^{(o)}} \operatorname{Ro}_{m}^{(4)}(c, \xi) \operatorname{So}_{m}(c, \eta) \operatorname{So}_{m}\left(c, \cos \varphi_{0}\right)\right]
\end{aligned}
$$

where the modal coefficients $a_{h, m}^{(e),(o)}$ are given by:

$$
\begin{aligned}
& a_{1, m}^{(e)}=a_{2, m}^{(e)}=-T \frac{\operatorname{Re}_{m}^{(1)}\left(c, \xi_{1}\right)}{\operatorname{Re}_{m}^{(4)}\left(c, \xi_{1}\right)} \\
& a_{1, m}^{(o)}=\zeta a_{2, m}^{(o)}=-\zeta T \frac{\operatorname{Ro}_{m}^{(1)}\left(c, \xi_{1}\right)}{\operatorname{Ro}_{m}^{(4)}\left(c, \xi_{1}\right)} .
\end{aligned}
$$

The normalized bistatic radar cross section (RCS) for an observation point in medium $1(0<\varphi<\pi)$ is:

$$
\begin{aligned}
\frac{\sigma_{\|, E}(\varphi)}{\lambda}=8 & \pi \mid \sum_{m=0}^{\infty}(-1)^{m}\left[\frac{a_{1, m}^{(e)}}{N_{m}^{(e)}} \operatorname{Se}_{m}(c, \cos \varphi) \operatorname{Se}_{m}\left(c, \cos \varphi_{0}\right)\right. \\
& \left.+\frac{a_{1, m}^{(o)}}{N_{m}^{(o)}} \operatorname{So}_{m}(c, \cos \varphi) \operatorname{So}_{m}\left(c, \cos \varphi_{0}\right)\right]\left.\right|^{2} \cdot(26)
\end{aligned}
$$

The magnetic field is given by

$$
\underline{H}_{h}=\frac{j}{c Z_{h} \sqrt{\xi^{2}-\eta^{2}}}\left(\frac{\partial E_{h z}}{\partial v} \hat{u}-\frac{\partial E_{h z}}{\partial u} \hat{v}\right),(h=1,2),
$$

and the electric current density on the surface $\xi=\xi_{1}$ of the metal cylinder is

$$
\underline{J}_{h}=\left.H_{h v}\right|_{\xi=\xi_{1}} \hat{z}
$$

where

$$
\begin{aligned}
\left.H_{h v}\right|_{\xi=\xi_{1}} & =\frac{T}{c Z_{h}} \sqrt{\frac{8 \pi}{\xi_{1}^{2}-\eta^{2}}} \sum_{m=0}^{\infty} j^{m}\left[\frac{\operatorname{Se}_{m}(c, \eta) \operatorname{Se}_{m}\left(c, \cos \varphi_{0}\right)}{N_{m}^{(e)} \operatorname{Re}_{m}^{(4)}\left(c, \xi_{1}\right)}\right. \\
& \left.+\zeta_{h} \frac{\operatorname{So}_{m}(c, \eta) \operatorname{So}_{m}\left(c, \cos \varphi_{0}\right)}{N_{m}^{(o)} \operatorname{Ro}_{m}^{(4)}\left(c, \xi_{1}\right)}\right]
\end{aligned}
$$

with

$$
\zeta_{1}=\zeta, \quad \zeta_{2}=1
$$

\section{B. Parallel configuration with H-polarization}

If the incident plane wave has a magnetic field $\underline{H}^{i}=\hat{z} H_{z}^{i}$ parallel to the z-axis with $H_{z}^{i}$ given by (12), then the total magnetic fields $H_{1 z}$ in medium 1 and $H_{2 z}$ in medium 2 may be written as

$$
\begin{aligned}
& H_{1 z}=H_{z}^{i}+H_{z}^{r}+H_{1 z}^{s}, \\
& H_{2 z}=H_{z}^{t}+H_{2 z}^{s},
\end{aligned}
$$

where the fields in the absence of the metal cylinder are:

$$
\begin{aligned}
& H_{z}^{i}+H_{z}^{r}= \\
& =\sqrt{8 \pi} \sum_{m=0}^{\infty} j^{m}\left[\frac{1-R}{N_{m}^{(e)}} \operatorname{Re}_{m}^{(1)}(c, \xi) \operatorname{Se}_{m}(c, \eta) \operatorname{Se}_{m}\left(c, \cos \varphi_{0}\right)\right. \\
& \left.+\frac{1+R}{N_{m}^{(o)}} \operatorname{Ro}_{m}^{(1)}(c, \xi) \operatorname{So}_{m}(c, \eta) \operatorname{So}_{m}\left(c, \cos \varphi_{0}\right)\right] \\
& H_{z}^{t}=\zeta T H_{z}^{i},
\end{aligned}
$$


whereas the scattered fields due to the presence of the cylinder are:

$$
\begin{aligned}
& H_{h z}^{s}= \\
& \sqrt{8 \pi} \sum_{m=0}^{\infty} j^{m}\left[\frac{b_{h, m}^{(e)}}{N_{m}^{(e)}} \operatorname{Re}_{m}^{(4)}(c, \xi) \operatorname{Se}_{m}(c, \eta) \operatorname{Se}_{m}\left(c, \cos \varphi_{0}\right)\right. \\
& \left.+\frac{b_{h, m}^{(o)}}{N_{m}^{(o)}} \operatorname{Ro}_{m}^{(4)}(c, \xi) \operatorname{So}_{m}(c, \eta) \operatorname{So}_{m}\left(c, \cos \varphi_{0}\right)\right],(h=1,2),
\end{aligned}
$$

with modal coefficients

$$
\begin{aligned}
& b_{1, m}^{(e)}=b_{2, m}^{(e)}=-\zeta T \frac{\operatorname{Re}_{m}^{(1)^{\prime}}\left(c, \xi_{1}\right)}{\operatorname{Re}_{m}^{(4)^{\prime}}\left(c, \xi_{1}\right)}, \\
& b_{1, m}^{(o)}=\zeta^{-1} b_{2, m}^{(o)}=-T \frac{\operatorname{Ro}_{m}^{(1)^{\prime}}\left(c, \xi_{1}\right)}{\operatorname{Ro}_{m}^{(4)^{\prime}}\left(c, \xi_{1}\right)} ;
\end{aligned}
$$

here and in the following, the prime on a radial function means derivative with respect to $\xi$.

The normalized bistatic RCS, $\sigma_{\|, H}(\varphi) / \lambda$ for an observation point in medium $1(0<\varphi<\pi)$ is given by (26) with $a_{1, m}^{(e),(o)}$ replaced by $b_{1, m}^{(e),(o)}$.

The electric surface current density on the metal cylinder is

$$
\underline{J}_{h}=-\left.H_{h z}\right|_{\xi=\xi_{1}} \hat{v},(h=1,2),
$$

where

$$
\begin{aligned}
& \left.H_{h z}\right|_{\xi=\xi_{1}}= \\
& -j T \frac{\zeta}{\zeta_{h}} \sqrt{\frac{8 \pi}{\xi_{1}^{2}-1}} \sum_{m=0}^{\infty} j^{m}\left[\zeta_{h} \frac{\operatorname{Se}_{m}(c, \eta) \operatorname{Se}_{m}\left(c, \cos \varphi_{0}\right)}{N_{m}^{(e)} \operatorname{Re}_{m}^{(4)^{\prime}}\left(c, \xi_{1}\right)}\right. \\
& \left.+\frac{\mathrm{So}_{m}(c, \eta) \mathrm{So}_{m}\left(c, \cos \varphi_{0}\right)}{N_{m}^{(o)} \operatorname{Ro}_{m}^{(4)^{\prime}}\left(c, \xi_{1}\right)}\right],
\end{aligned}
$$

with $\zeta_{h}$ given by (30).

\section{Perpendicular configuration with E-polarization}

With reference to Fig. 2, the incident electric field $E_{z}^{i}$ is still given by (12), and the total fields $E_{1 z}$ in $x>0$ and $E_{2 z}$ in $x<0$ by (19) and (20).

In the absence of the metal cylinder,

$$
\begin{aligned}
& E_{z}^{i}+E_{z}^{r}=\sqrt{8 \pi} \times \\
& \sum_{m=0}^{\infty} j^{m}\left[\frac{1+R(-1)^{m}}{N_{m}^{(e)}} \operatorname{Re}_{m}^{(1)}(c, \xi) \operatorname{Se}_{m}(c, \eta) \operatorname{Se}_{m}\left(c, \cos \varphi_{0}\right)\right. \\
+ & \left.\frac{1-R(-1)^{m}}{N_{m}^{(o)}} \operatorname{Ro}_{m}^{(1)}(c, \xi) \operatorname{So}_{m}(c, \eta) \operatorname{So}_{m}\left(c, \cos \varphi_{0}\right)\right], \quad \text { (40) }
\end{aligned}
$$

and $E_{z}^{t}$ is given by (22), whereas the scattered fields due to the presence of the cylinder are

$$
\begin{gathered}
E_{h z}^{s}= \\
\sqrt{8 \pi} \sum_{m=0}^{\infty} j^{m}\left[\frac{c_{h, m}^{(e)}}{N_{m}^{(e)}} \operatorname{Re}_{m}^{(4)}(c, \xi) \operatorname{Se}_{m}(c, \eta) \operatorname{Se}_{m}\left(c, \cos \varphi_{0}\right)\right. \\
\left.+\frac{c_{h, m}^{(o)}}{N_{m}^{(o)}} \operatorname{Ro}_{m}^{(4)}(c, \xi) \operatorname{So}_{m}(c, \eta) \operatorname{So}_{m}\left(c, \cos \varphi_{0}\right)\right],(h=1,2),
\end{gathered}
$$

where

$$
\begin{gathered}
c_{1,2 \ell}^{(e)}=c_{2,2 \ell}^{(e)}=-T \frac{\operatorname{Re}_{2 \ell}^{(1)}\left(c, \xi_{1}\right)}{\operatorname{Re}_{2 \ell}^{(4)}\left(c, \xi_{1}\right)}, \\
c_{1,2 \ell+1}^{(e)}=\zeta c_{2,2 \ell+1}^{(e)}=-\zeta T \frac{\operatorname{Re}_{2 \ell+1}^{(1)}\left(c, \xi_{1}\right)}{\operatorname{Re}_{2 \ell+1}^{(4)}\left(c, \xi_{1}\right)}, \\
c_{1,2 \ell}^{(o)}=\zeta c_{2,2 \ell}^{(o)}=-\zeta T \frac{\operatorname{Ro}_{2 \ell}^{(1)}\left(c, \xi_{1}\right)}{\operatorname{Ro}_{2 \ell}^{(4)}\left(c, \xi_{1}\right)}, \\
c_{1,2 \ell+1}^{(o)}=c_{2,2 \ell+1}^{(o)}=-T \frac{\operatorname{Ro}_{2 \ell+1}^{(1)}\left(c, \xi_{1}\right)}{\operatorname{Ro}_{2 \ell+1}^{(4)}\left(c, \xi_{1}\right)},
\end{gathered}
$$

with $\ell=0,1,2, \ldots$ a non-negative integer.

The normalized bistatic RCS, $\sigma_{\perp, E}(\varphi) / \lambda$ for an observation point in medium $1(-\pi / 2<\varphi<\pi / 2)$ is given by (26) with $a_{1, m}^{(e),(o)}$ replaced by $c_{1, m}^{(e),(o)}$.

The electric current density on the surface of the metal cylinder is given by (28) where $\left.H_{2 v}\right|_{\xi=\xi_{1}}$ is still given by (29) with $h=2$, whereas

$$
\begin{aligned}
\left.H_{1 v}\right|_{\xi=\xi_{1}}=\frac{T}{c Z_{1}} & \sqrt{\frac{8 \pi}{\xi_{1}^{2}-\eta^{2}} \times} \\
\sum_{l=0}^{\infty}(-1)^{\ell} & {\left[\frac{\operatorname{Se}_{2 \ell}(c, \eta) \operatorname{Se}_{2 \ell}\left(c, \cos \varphi_{0}\right)}{N_{2 \ell}^{(e)} \operatorname{Re}_{2 \ell}^{(4)}\left(c, \xi_{1}\right)}\right.} \\
& +j \zeta \frac{\operatorname{Se}_{2 \ell+1}(c, \eta) \operatorname{Se}_{2 \ell+1}\left(c, \cos \varphi_{0}\right)}{N_{2 \ell+1}^{(e)} \operatorname{Re}_{2 \ell+1}^{(4)}\left(c, \xi_{1}\right)} \\
& +\zeta \frac{\operatorname{So}_{2 \ell}(c, \eta) \operatorname{So}_{2 \ell}\left(c, \cos \varphi_{0}\right)}{N_{2 \ell}^{(o)} \operatorname{Ro}_{2 \ell}^{(4)}\left(c, \xi_{1}\right)} \\
& \left.j \frac{\operatorname{So}_{2 \ell+1}(c, \eta) \operatorname{So}_{2 \ell+1}\left(c, \cos \varphi_{0}\right)}{N_{2 \ell+1}^{(o)} \operatorname{Ro}_{2 \ell+1}^{(4)}\left(c, \xi_{1}\right)}\right] .
\end{aligned}
$$

\section{Perpendicular configuration with H-polarization}

The incident magnetic field $H_{z}^{i}$ is given by (12), and the total magnetic fields $H_{1 z}$ in $x>0$ and $H_{2 z}$ in $x<0$ by (31) and (32) (see Fig. 2). In the absence of the metal cylinder,

$$
\begin{aligned}
& H_{z}^{i}+H_{z}^{r}= \\
& \sqrt{8 \pi} \sum_{m=0}^{\infty} j^{m}\left[\frac{1-R(-1)^{m}}{N_{m}^{(e)}} \operatorname{Re}_{m}^{(1)}(c, \xi) \operatorname{Se}_{m}(c, \eta) \operatorname{Se}_{m}\left(c, \cos \varphi_{0}\right)\right. \\
& \left.+\frac{1+R(-1)^{m}}{N_{m}^{(o)}} \operatorname{Ro}_{m}^{(1)}(c, \xi) \operatorname{So}_{m}(c, \eta) \operatorname{So}_{m}\left(c, \cos \varphi_{0}\right)\right]
\end{aligned}
$$

and $H_{z}^{t}$ is given by (34); the scattered fields due to the presence of the cylinder are

$$
\begin{aligned}
& H_{h z}^{s}=\sqrt{8 \pi} \sum_{m=0}^{\infty} j^{m}\left[\frac{d_{h, m}^{(e)}}{N_{m}^{(e)}} \operatorname{Re}_{m}^{(4)}(c, \xi) \operatorname{Se}_{m}(c, \eta) \operatorname{Se}_{m}\left(c, \cos \varphi_{0}\right)\right. \\
& \left.+\frac{d_{h, m}^{(o)}}{N_{m}^{(o)}} \operatorname{Ro}_{m}^{(4)}(c, \xi) \operatorname{So}_{m}(c, \eta) \operatorname{So}_{m}\left(c, \cos \varphi_{0}\right)\right]
\end{aligned}
$$


where

$$
\begin{gathered}
d_{1,2 \ell}^{(e)}=\quad d_{2,2 \ell}^{(e)}=-\zeta T \frac{\operatorname{Re}_{2 \ell}^{(1)^{\prime}}\left(c, \xi_{1}\right)}{\operatorname{Re}_{2 \ell}^{(4)^{\prime}}\left(c, \xi_{1}\right)} \\
d_{1,2 \ell+1}^{(e)}=\zeta^{-1} d_{2,2 \ell+1}^{(e)}=-T \frac{\operatorname{Re}_{2 \ell+1}^{(1)^{\prime}}\left(c, \xi_{1}\right)}{\operatorname{Re}_{2 \ell+1}^{(4)^{\prime}}\left(c, \xi_{1}\right)}, \\
d_{1,2 \ell}^{(o)}=\zeta^{-1} d_{2,2 \ell}^{(o)}=-T \frac{\operatorname{Ro}_{2 \ell}^{(1)^{\prime}}\left(c, \xi_{1}\right)}{\operatorname{Ro}_{2 \ell}^{(4)^{\prime}}\left(c, \xi_{1}\right)} \\
d_{1,2 \ell+1}^{(o)}=\quad d_{2,2 \ell+1}^{(o)}=-\zeta T \frac{\operatorname{Ro}_{2 \ell+1}^{(1)^{\prime}}\left(c, \xi_{1}\right)}{\operatorname{Ro}_{2 \ell+1}^{(4)^{\prime}}\left(c, \xi_{1}\right)},
\end{gathered}
$$

The normalized bistatic RCS, $\sigma_{\perp, H}(\varphi) / \lambda$ for an observation point in medium $1(-\pi / 2<\varphi<\pi / 2)$ is given by (26) with $a_{1, m}^{(e),(o)}$ replaced by $d_{1, m}^{(e),(o)}$.

The surface current density on the metal cylinder is given by (38) where $\left.H_{2 z}\right|_{\xi=\xi_{1}}$ is still given by (39) with $h=2$, whereas

$$
\begin{aligned}
&\left.H_{1 z}\right|_{\xi=\xi_{1}}=T \sqrt{\frac{8 \pi}{\xi_{1}^{2}-1} \times} \\
& \sum_{l=0}^{\infty}(-1)^{\ell}\left[-j \zeta \frac{\mathrm{Se}_{2 \ell}(c, \eta) \operatorname{Se}_{2 \ell}\left(c, \cos \varphi_{0}\right)}{N_{2 \ell}^{(e)} \operatorname{Re}_{2 \ell}^{(4)^{\prime}}\left(c, \xi_{1}\right)}\right. \\
&+\frac{\operatorname{Se}_{2 \ell+1}(c, \eta) \operatorname{Se}_{2 \ell+1}\left(c, \cos \varphi_{0}\right)}{N_{2 \ell+1}^{(e)} \operatorname{Re}_{2 \ell+1}^{(4)^{\prime}}\left(c, \xi_{1}\right)} \\
&-j \frac{\operatorname{So}_{2 \ell}(c, \eta) \operatorname{So}_{2 \ell}\left(c, \cos \varphi_{0}\right)}{N_{2 \ell}^{(o)} \operatorname{Ro}_{2 \ell}^{(4)^{\prime}}\left(c, \xi_{1}\right)} \\
& \\
&\left.\zeta \frac{\operatorname{So}_{2 \ell+1}(c, \eta) \operatorname{So}_{2 \ell+1}\left(c, \cos \varphi_{0}\right)}{N_{2 \ell+1}^{(o)} \operatorname{Ro}_{2 \ell+1}^{(4)^{\prime}}\left(c, \xi_{1}\right)}\right] .
\end{aligned}
$$

\section{LINE SOURCE INCIDENCE}

\section{A. Parallel configuration with electric line source}

For an isotropic electric line source that generates an electric field $\hat{z} E_{z}^{i}$ with $E_{z}^{i}$ given by (13), the total electric fields $E_{1 z}$ in medium 1 and $E_{2 z}$ in medium 2 of Fig. 1 are still given by (19) and (20), where now the reflected and transmitted fields in the absence of the metal cylinder are

$$
E_{z}^{r}=R H_{0}^{(2)}(k \tilde{r}), \quad E_{z}^{t}=T E_{z}^{i},
$$

where

$$
\tilde{r}=\sqrt{\left(x-x_{0}\right)^{2}+\left(y+y_{0}\right)^{2}}
$$

is the distance from the image line source at $\left(x_{0},-y_{0}\right)$ to the observation point. Consequently,

$$
\begin{aligned}
& E_{z}^{i}+E_{z}^{r}= \\
& 4 \sum_{m=0}^{\infty}\left[\frac{1+R}{N_{m}^{(e)}} \operatorname{Re}_{m}^{(1)}\left(c, \xi_{<}\right) \operatorname{Re}_{m}^{(4)}\left(c, \xi_{>}\right) \operatorname{Se}_{m}\left(c, \eta_{0}\right) \operatorname{Se}_{m}(c, \eta)+\right. \\
& \left.\frac{1-R}{N_{m}^{(o)}} \operatorname{Ro}_{m}^{(1)}\left(c, \xi_{<}\right) \operatorname{Ro}_{m}^{(4)}\left(c, \xi_{>}\right) \operatorname{So}_{m}\left(c, \eta_{0}\right) \operatorname{So}_{m}(c, \eta)\right]
\end{aligned}
$$

The scattered fields due to the presence of the cylinder are

$$
\begin{aligned}
& E_{h z}^{s}= \\
& 4 \sum_{m=0}^{\infty}\left[\frac{a_{h, m}^{(e)}}{N_{m}^{(e)}} \operatorname{Re}_{m}^{(4)}\left(c, \xi_{0}\right) \operatorname{Re}_{m}^{(4)}(c, \xi) \operatorname{Se}_{m}\left(c, \eta_{0}\right) \operatorname{Se}_{m}(c, \eta)+\right. \\
& \left.\frac{a_{h, m}^{(o)}}{N_{m}^{(o)}} \operatorname{Ro}_{m}^{(4)}\left(c, \xi_{0}\right) \operatorname{Ro}_{m}^{(4)}(c, \xi) \operatorname{So}_{m}\left(c, \eta_{0}\right) \operatorname{So}_{m}(c, \eta)\right],(h=1,2),
\end{aligned}
$$

where $a_{h, m}^{(e),(o)}$ are given by (24),(25).

The surface current density on the metal cylinder is given by (28) with:

$$
\begin{aligned}
& \left.H_{h v}\right|_{\xi=\xi_{1}}= \\
& \frac{4 T}{c Z_{h} \sqrt{\xi_{1}^{2}-\eta^{2}}} \sum_{m=0}^{\infty}\left[\frac{\operatorname{Re}_{m}^{(4)}\left(c, \xi_{0}\right)}{N_{m}^{(e)} \operatorname{Re}_{m}^{(4)}\left(c, \xi_{1}\right)} \operatorname{Se}_{m}(c, \eta) \operatorname{Se}_{m}\left(c, \eta_{0}\right)\right. \\
& \left.+\zeta_{h} \frac{\operatorname{Ro}_{m}^{(4)}\left(c, \xi_{0}\right)}{N_{m}^{(o)} \operatorname{Ro}_{m}^{(4)}\left(c, \xi_{1}\right)} \mathrm{So}_{m}(c, \eta) \operatorname{So}_{m}\left(c, \eta_{0}\right)\right],(h=1,2) .
\end{aligned}
$$

\section{B. Parallel configuration with magnetic line source}

For an isotropic line source that generates a magnetic field $\hat{z} H_{z}^{i}$ with $H_{z}^{i}$ given by (13), the total magnetic fields $H_{1 z}$ in medium 1 and $H_{2 z}$ in medium 2 of Fig. 1 are still given by (31) and (32), where now the reflected and transmitted fields in the absence of the metal cylinder are

$$
H_{z}^{r}=-R H_{0}^{(2)}(k \tilde{r}), \quad H_{z}^{t}=\zeta T H_{z}^{i},
$$

with $\tilde{r}$ given by (55). Consequently,

$$
\begin{aligned}
& H_{z}^{i}+H_{z}^{r}= \\
& 4 \sum_{m=0}^{\infty}\left[\frac{1-R}{N_{m}^{(e)}} \operatorname{Re}_{m}^{(1)}\left(c, \xi_{<}\right) \operatorname{Re}_{m}^{(4)}\left(c, \xi_{>}\right) \operatorname{Se}_{m}\left(c, \eta_{0}\right) \operatorname{Se}_{m}(c, \eta)+\right. \\
& \left.\frac{1+R}{N_{m}^{(o)}} \operatorname{Ro}_{m}^{(1)}\left(c, \xi_{<}\right) \operatorname{Ro}_{m}^{(4)}\left(c, \xi_{>}\right) \operatorname{So}_{m}\left(c, \eta_{0}\right) \operatorname{So}_{m}(c, \eta)\right]
\end{aligned}
$$

The scattered fields due to the presence of the cylinder are

$H_{h z}^{s}=$

$4 \sum_{m=0}^{\infty}\left[\frac{b_{h, m}^{(e)}}{N_{m}^{(e)}} \operatorname{Re}_{m}^{(4)}\left(c, \xi_{0}\right) \operatorname{Re}_{m}^{(4)}(c, \xi) \operatorname{Se}_{m}\left(c, \eta_{0}\right) \operatorname{Se}_{m}(c, \eta)+\right.$

$\left.\frac{b_{h, m}^{(o)}}{N_{m}^{(o)}} \operatorname{Ro}_{m}^{(4)}\left(c, \xi_{0}\right) \operatorname{Ro}_{m}^{(4)}(c, \xi) \operatorname{So}_{m}\left(c, \eta_{0}\right) \operatorname{So}_{m}(c, \eta)\right],(h=1,2)$,

where $b_{h, m}^{(e),(o)}$ are given by (36), (37).

The surface current density on the metal cylinder is given by (38) with: 


$$
\begin{aligned}
& \left.H_{h z}\right|_{\xi=\xi_{1}}=\frac{-4 j T}{\sqrt{\xi_{1}^{2}-1}} \frac{\zeta}{\zeta_{h}} \times \\
& \sum_{m=0}^{\infty}\left[\zeta_{h} \frac{\operatorname{Re}_{m}^{(4)}\left(c, \xi_{0}\right)}{N_{m}^{(e)} \operatorname{Re}_{m}^{(4)^{\prime}}\left(c, \xi_{1}\right)} \operatorname{Se}_{m}(c, \eta) \operatorname{Se}_{m}\left(c, \eta_{0}\right)+\right. \\
& \left.\frac{\operatorname{Ro}_{m}^{(4)}\left(c, \xi_{0}\right)}{N_{m}^{(o)} \operatorname{Ro}_{m}^{(4)^{\prime}}\left(c, \xi_{1}\right)} \operatorname{So}_{m}(c, \eta) \operatorname{So}_{m}\left(c, \eta_{0}\right)\right],(h=1,2) .
\end{aligned}
$$

\section{Perpendicular configuration with electric line source}

An isotropic electric line source that generates an incident electric field parallel to the z-axis with $E_{z}^{i}$ given by (13), produces total electric fields $E_{1 z}$ in medium 1 and $E_{2 z}$ in medium 2 of Fig. 2 that are given by (19) and (20). The reflected and transmitted fields in the absence of the metal cylinder are

$$
E_{z}^{r}=R H_{0}^{(2)}(k \tilde{\tilde{r}}), \quad E_{z}^{t}=T E_{z}^{i}
$$

where

$$
\tilde{\tilde{r}}=\sqrt{\left(x+x_{0}\right)^{2}+\left(y-y_{0}\right)^{2}}
$$

is the distance of the image line source located at $\left(-x_{0}, y_{0}\right)$ from the observation point. Consequently,

$$
\begin{aligned}
& E_{z}^{i}+E_{z}^{r}=4 \sum_{m=0}^{\infty}\left[\frac{1+R(-1)^{m}}{N_{m}^{(e)}} \operatorname{Re}_{m}^{(1)}\left(c, \xi_{<}\right) \operatorname{Re}_{m}^{(4)}\left(c, \xi_{>}\right) \times\right. \\
& \operatorname{Se}_{m}\left(c, \eta_{0}\right) \operatorname{Se}_{m}(c, \eta)+ \\
& \left.\frac{1-R(-1)^{m}}{N_{m}^{(o)}} \operatorname{Ro}_{m}^{(1)}\left(c, \xi_{<}\right) \operatorname{Ro}_{m}^{(4)}\left(c, \xi_{>}\right) \operatorname{So}_{m}\left(c, \eta_{0}\right) \operatorname{So}_{m}(c, \eta)\right] .
\end{aligned}
$$

The scattered fields due to the presence of the cylinder are:

$E_{h z}^{s}=$

$4 \sum_{m=0}^{\infty}\left[\frac{c_{h, m}^{(e)}}{N_{m}^{(e)}} \operatorname{Re}_{m}^{(4)}\left(c, \xi_{0}\right) \operatorname{Re}_{m}^{(4)}(c, \xi) \operatorname{Se}_{m}\left(c, \eta_{0}\right) \operatorname{Se}_{m}(c, \eta)+\right.$
$\left.\frac{c_{h, m}^{(o)}}{N_{m}^{(o)}} \operatorname{Ro}_{m}^{(4)}\left(c, \xi_{0}\right) \operatorname{Ro}_{m}^{(4)}(c, \xi) \operatorname{So}_{m}\left(c, \eta_{0}\right) \operatorname{So}_{m}(c, \eta)\right],(h=1,2)$,

where $c_{h, m}^{(e),(o)}$ are given by (42)-(45).

The surface current density on the metal cylinder is given by (28) with:

$$
\begin{aligned}
& \left.H_{1 v}\right|_{\xi=\xi_{1}}=\frac{4}{c Z_{1} \sqrt{\xi_{1}^{2}-\eta^{2}}} \times \\
& \sum_{m=0}^{\infty}\left[\frac{1+R(-1)^{m}}{N_{m}^{(e)}} \frac{\operatorname{Re}_{m}^{(4)}\left(c, \xi_{0}\right)}{\operatorname{Re}_{m}^{(4)}\left(c, \xi_{1}\right)} \operatorname{Se}_{m}(c, \eta) \operatorname{Se}_{m}\left(c, \eta_{0}\right)\right. \\
& \left.+\frac{1-R(-1)^{m}}{N_{m}^{(o)}} \frac{\operatorname{Ro}_{m}^{(4)}\left(c, \xi_{0}\right)}{\operatorname{Ro}_{m}^{(4)}\left(c, \xi_{1}\right)} \mathrm{So}_{m}(c, \eta) \mathrm{So}_{m}\left(c, \eta_{0}\right)\right],
\end{aligned}
$$

$$
\begin{aligned}
& \left.H_{2 v}\right|_{\xi=\xi_{1}}=\frac{4 T}{c Z_{2} \sqrt{\xi_{1}^{2}-\eta^{2}}} \times \\
& \sum_{m=0}^{\infty}\left[\frac{\operatorname{Re}_{m}^{(4)}\left(c, \xi_{0}\right)}{N_{m}^{(e)} \operatorname{Re}_{m}^{(4)}\left(c, \xi_{1}\right)} \operatorname{Se}_{m}(c, \eta) \operatorname{Se}_{m}\left(c, \eta_{0}\right)\right. \\
& \left.+\frac{\operatorname{Ro}_{m}^{(4)}\left(c, \xi_{0}\right)}{N_{m}^{(o)} \operatorname{Ro}_{m}^{(4)}\left(c, \xi_{1}\right)} \operatorname{So}_{m}(c, \eta) \operatorname{So}_{m}\left(c, \eta_{0}\right)\right] .
\end{aligned}
$$

\section{Perpendicular configuration with magnetic line source}

For an isotropic line source that generates a magnetic filed parallel to the z-axis with $H_{z}^{i}$ given by (13), the total magnetic fields $H_{1 z}$ in medium 1 and $H_{2 z}$ in medium 2 of Fig. 2 are given by (31) and (32) where the reflected and transmitted fields in the absence of the metal cylinder are

$$
H_{z}^{r}=-R H_{0}^{(2)}(k \tilde{\tilde{r}}), \quad H_{z}^{t}=\zeta T H_{z}^{i}
$$

with $\tilde{\tilde{r}}$ given by (64). Consequently,

$$
\begin{aligned}
& H_{z}^{i}+H_{z}^{r}=4 \times \sum_{m=0}^{\infty} \\
& {\left[\frac{1-R(-1)^{m}}{N_{m}^{(e)}} \operatorname{Re}_{m}^{(1)}\left(c, \xi_{<}\right) \operatorname{Re}_{m}^{(4)}\left(c, \xi_{>}\right) \operatorname{Se}_{m}\left(c, \eta_{0}\right) \operatorname{Se}_{m}(c, \eta)+\right.} \\
& \left.\frac{1+R(-1)^{m}}{N_{m}^{(o)}} \operatorname{Ro}_{m}^{(1)}\left(c, \xi_{<}\right) \operatorname{Ro}_{m}^{(4)}\left(c, \xi_{>}\right) \operatorname{So}_{m}\left(c, \eta_{0}\right) \operatorname{So}_{m}(c, \eta)\right] .
\end{aligned}
$$

The scattered fields due to the presence of the cylinder are $H_{h z}^{s}=$

$$
\begin{aligned}
& 4 \sum_{m=0}^{\infty}\left[\frac{d_{h, m}^{(e)}}{N_{m}^{(e)}} \operatorname{Re}_{m}^{(4)}\left(c, \xi_{0}\right) \operatorname{Re}_{m}^{(4)}(c, \xi) \operatorname{Se}_{m}\left(c, \eta_{0}\right) \operatorname{Se}_{m}(c, \eta)+\right. \\
& \left.\frac{d_{h, m}^{(o)}}{N_{m}^{(o)}} \operatorname{Ro}_{m}^{(4)}\left(c, \xi_{0}\right) \operatorname{Ro}_{m}^{(4)}(c, \xi) \operatorname{So}_{m}\left(c, \eta_{0}\right) \operatorname{So}_{m}(c, \eta)\right],(h=1,2),
\end{aligned}
$$

where $d_{h, m}^{(e),(o)}$ are given by (49)-(52).

The surface current density on the metal cylinder is given by (38) with

$$
\begin{gathered}
\left.H_{1 z}\right|_{\xi=\xi_{1}}=\frac{-4 j}{\sqrt{\xi_{1}^{2}-1}} \times \\
\sum_{m=0}^{\infty}\left[\frac{1-R(-1)^{m}}{N_{m}^{(e)}} \frac{\operatorname{Re}_{m}^{(4)}\left(c, \xi_{0}\right)}{\operatorname{Re}_{m}^{(4)^{\prime}}\left(c, \xi_{1}\right)} \operatorname{Se}_{m}(c, \eta) \operatorname{Se}_{m}\left(c, \eta_{0}\right)\right. \\
\left.+\frac{1+R(-1)^{m}}{N_{m}^{(o)}} \frac{\operatorname{Ro}_{m}^{(4)}\left(c, \xi_{0}\right)}{\operatorname{Ro}_{m}^{(4)^{\prime}}\left(c, \xi_{1}\right)} \operatorname{So}_{m}(c, \eta) \operatorname{So}_{m}\left(c, \eta_{0}\right)\right] \\
\left.H_{2 z}\right|_{\xi=\xi_{1}}=\frac{-4 j \zeta T}{\sqrt{\xi_{1}^{2}-1}} \times \\
\sum_{m=0}^{\infty}\left[\frac{\operatorname{Re}_{m}^{(4)}\left(c, \xi_{0}\right)}{N_{m}^{(e)} \operatorname{Re}_{m}^{(4)^{\prime}}\left(c, \xi_{1}\right)} \operatorname{Se}_{m}(c, \eta) \operatorname{Se}_{m}\left(c, \eta_{0}\right)\right. \\
\left.+\frac{\operatorname{Ro}_{m}^{(4)}\left(c, \xi_{0}\right)}{N_{m}^{(o)} \operatorname{Ro}_{m}^{(4)^{\prime}}\left(c, \xi_{1}\right)} \operatorname{So}_{m}(c, \eta) \operatorname{So}_{m}\left(c, \eta_{0}\right)\right]
\end{gathered}
$$




\section{THE PARTICULAR CASE OF THE STRIP}

In the limit case of the metal strip, $\xi_{1}$, the minor axis of the elliptic cylinder's cross section shrinks to zero and the major axis equals the interfocal distance $d$. Thus, the formulas obtained in the previous sections apply to the case of a metal strip of width $d$ that is either on the interface between the two media, or perpendicular to the interface and protruding a width $d / 2$ into each medium, when one sets $\xi_{1}=1$ ( or $u_{1}=0$ ).

By using the relations

$$
\operatorname{Ro}_{m}^{(1)}(c, 1)=0,\left.\quad \frac{\partial}{\partial u} \operatorname{Re}_{m}^{(1)}(c, \xi)\right|_{u=0}=0
$$

it is seen that

$$
\begin{aligned}
& \left.a_{h, m}^{(o)}\right|_{\xi_{1}=1}=0,\left.\quad b_{h, m}^{(e)}\right|_{\xi_{1}=1}=0, \\
& \left.c_{h, m}^{(o)}\right|_{\xi_{1}=1}=0,\left.\quad d_{h, m}^{(e)}\right|_{\xi_{1}=1}=0, \quad(h=1,2)
\end{aligned}
$$

For the H-polarization cases, it is useful to observe that in formulas such as (39), (53), (62), (72) and (73) one may perform the substitution:

$$
\sqrt{\xi_{1}^{2}-1} \operatorname{Re},\left.\mathrm{o}^{(4)^{\prime}}\left(c, \xi_{1}\right)\right|_{\xi_{1}=1}=\frac{\partial}{\partial u} \operatorname{Re},\left.\mathrm{o}_{m}^{(4)}(c, \xi)\right|_{u=0} .
$$

\section{NUMERICAL RESUlts}

Numerical results are provided for bistatic radar cross sections, induced currents and the particular case of the strip derived in the previous sections. The computations of the Mathieu functions are performed using software that is based, in part, on some of the Fortran subroutines provided in [16]. However, since the subroutines in [16] apply the GoldsteinInce normalization [17],[18],[19], they were modified to account for the Stratton-Chu normalization [14],[15] used in this work. Additionally, the subroutines taken from [16] were translated to Fortran 90 so that computations at quadruple precision could be run to verify the results. For all the series involved in the following results, convergence was achieved within the first 50 terms. In particular, each curve related to a bistatic RCS was evaluated at 181 points, whereas the polar plots for the induced currents were evaluated at 360 points. The computation time for each figure on a personal computer with a CPU that runs at $1.5 \mathrm{GHz}$ is about one minute.

In all the figures that follow (with the exception of the results for the strip), the same elliptic cross-section with size $\xi_{1}=2$ is used for both the parallel and perpendicular configurations of Fig. 1 and 2, respectively.

Results are presented for various values of the parameter $c$ given in (8), so that different cases for the size of the crosssection versus the wavelength are examined. The effect of different material is also accounted for by showing results for different values of $\zeta$ given by (10).

All results related to the plane wave incidence correspond to a field given by (12) that implies an amplitude of $1 \mathrm{~V} / \mathrm{m}$ in the electric case or $1 \mathrm{~A} / \mathrm{m}$ in the magnetic case.

The first four figures deal with the bistatic RCS for an elliptic cylinder with size $\xi_{1}=2$.
Fig. 3 shows the bistatic radar cross section, given by (26), for the parallel configuration of Fig. 1, due to an E-polarized plane wave incident at an angle $\varphi_{0}=\pi / 4$ when $\zeta=1 / 2$. It is observed that the RCS is larger and shows a more complex behavior with increasing values of $c$. Fig. 4 shows the bistatic radar cross section, for the parallel configuration of Fig. 1, due to an H-polarized plane wave incident at an angle $\varphi_{0}=\pi / 4$ when $\zeta=1 / 2$. This behavior is similar to the one of Fig. 3. Fig. 5 shows the bistatic radar cross section, for the perpendicular configuration of Fig. 2, due to an Epolarized plane wave incident at an angle $\varphi_{0}=\pi / 4$ when $\zeta=1 / 2$. Fig. 6 shows the bistatic radar cross section, for the perpendicular configuration of Fig. 2, due to an H-polarized plane wave incident at an angle $\varphi_{0}=\pi / 4$ when $\zeta=1 / 2$.

The following four figures deal with the current induced over the surface of a PEC cylinder with size $\xi_{1}=2$ and due to plane wave incidence. Fig. 7 shows the magnitude of the current density $J_{z}$ for the parallel configuration due to an Epolarized plane wave incident at $\varphi_{0}=\pi / 6$ when $\zeta=1 / 3$. There is a jump in the value of $\left|J_{z}\right|$ at $v=0$ and $v=\pi$, which is due to the transition from medium 1 to medium 2 , as apparent from (29). 


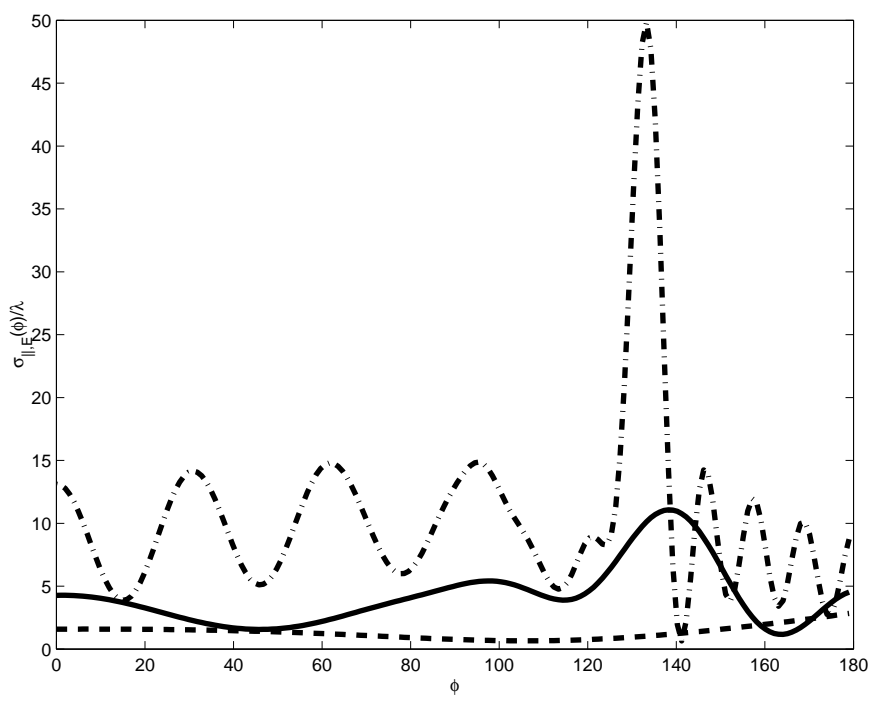

Fig. 3. Bistatic RCS for the parallel configuration when the source is an E-polarized plane wave incident at an angle $\varphi_{0}=\pi / 4$ and $\zeta=1 / 2$. Results correspond to $c=1$ (dashed line), $c=\pi$ (solid line); $c=10$ (dash-dot line).

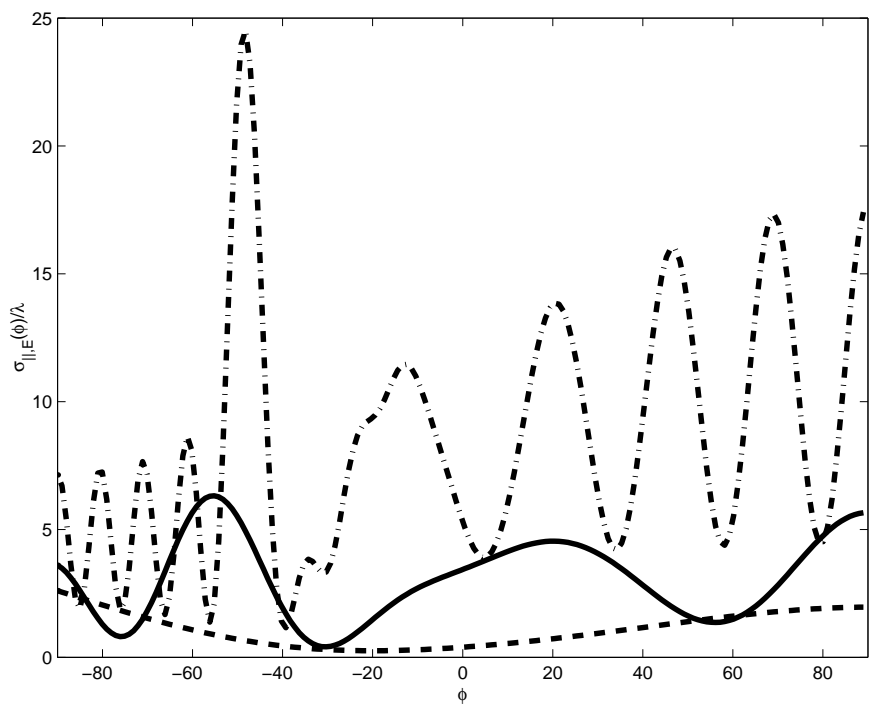

Fig. 5. Bistatic RCS for the perpendicular configuration when the source is an E-polarized plane wave incident at an angle $\varphi_{0}=\pi / 4$ and $\zeta=1 / 2$. Results correspond to $c=1$ (dashed line), $c=\pi$ (solid line); $c=10$ (dash-dot line).

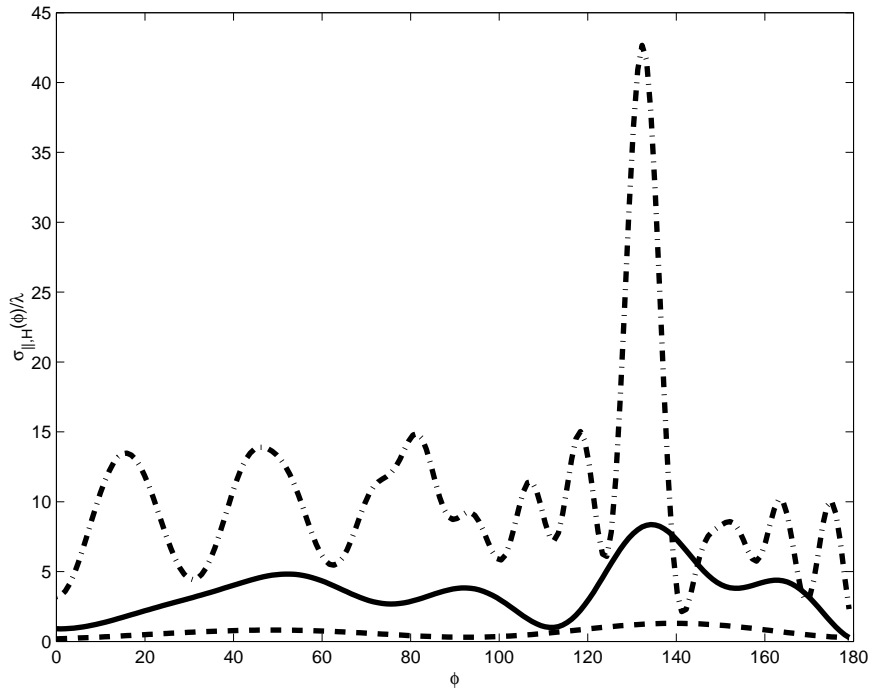

Fig. 4. Bistatic RCS for the parallel configuration when the source is an H-polarized plane wave incident at an angle $\varphi_{0}=\pi / 4$ and $\zeta=1 / 2$. Results correspond to $c=1$ (dashed line), $c=\pi$ (solid line); $c=10$ (dash-dot line).

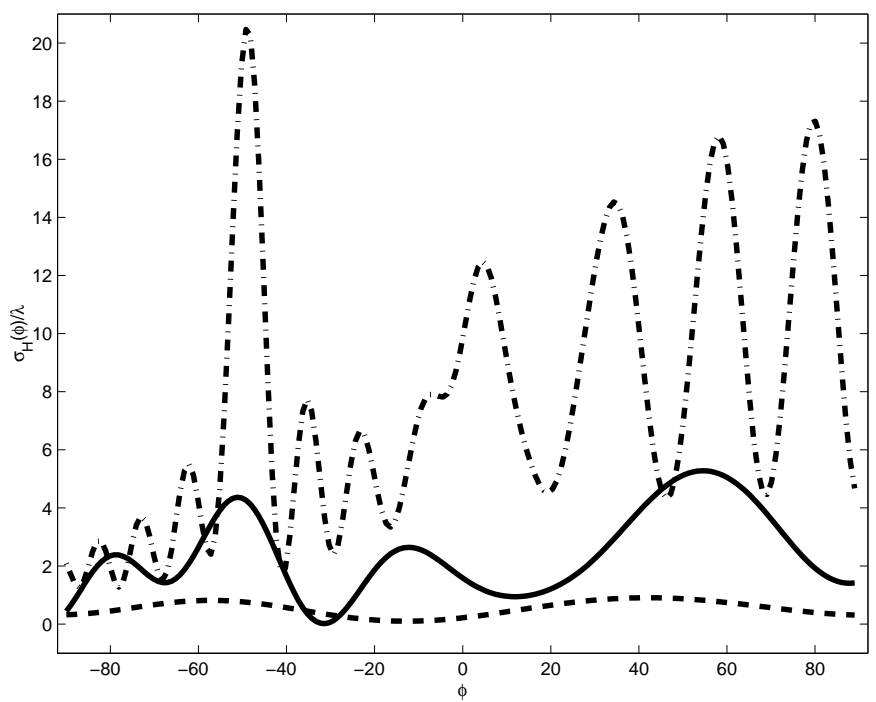

Fig. 6. Bistatic RCS for the perpendicular configuration when the source is an $\mathrm{H}$-polarized plane wave incident at an angle $\varphi_{0}=\pi / 4$ and $\zeta=1 / 2$. Results correspond to $c=1$ (dashed line), $c=\pi$ (solid line); $c=10$ (dash-dot line). 


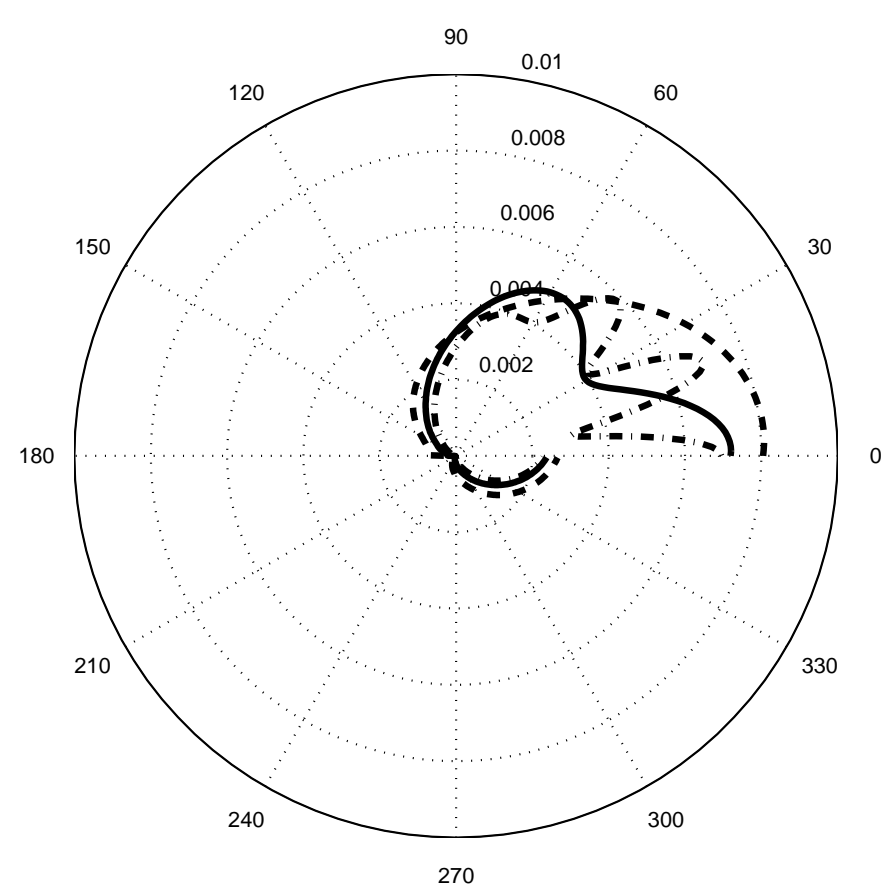

Fig. 7. Polar plot of the current density $\left|J_{z}\right|$ induced over the surface of an elliptic cylinder with $\xi_{1}=2$ for the parallel configuration when the source is an E-polarized plane wave incident at $\varphi_{0}=\pi / 6$ and $\zeta=1 / 3$. Results correspond to $c=1$ (dashed line), $c=\pi$ (solid line); $c=10$ (dash-dot line). Observe the symmetry and the jump at $v=0, \pi$ due to $\zeta \neq 1$ at the interface from medium 1 and 2.

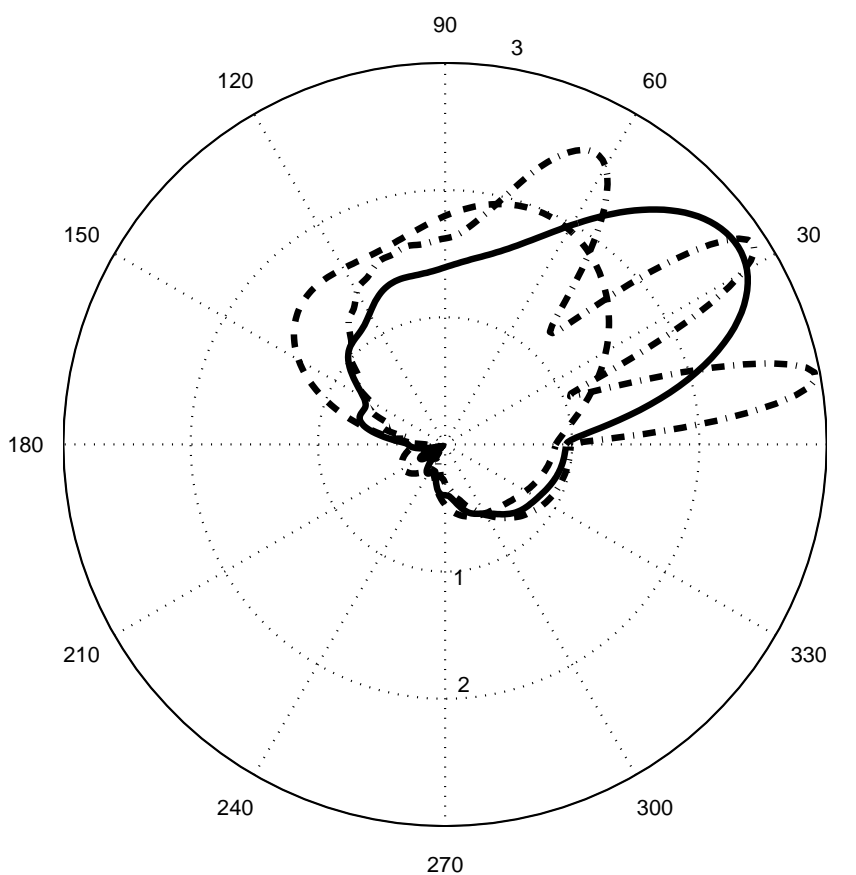

Fig. 8. Polar plot of the current density $\left|J_{v}\right|$ induced over the surface of an elliptic cylinder with $\xi_{1}=2$ for the parallel configuration when the source is an $\mathrm{H}$-polarized plane wave incident at $\varphi_{0}=\pi / 6$ and $\zeta=1 / 3$. Results correspond to $c=1$ (dashed line), $c=\pi$ (solid line); $c=10$ (dash-dot line). Observe the continuity at $v=0, \pi$ at the interface between medium 1 and 2 .

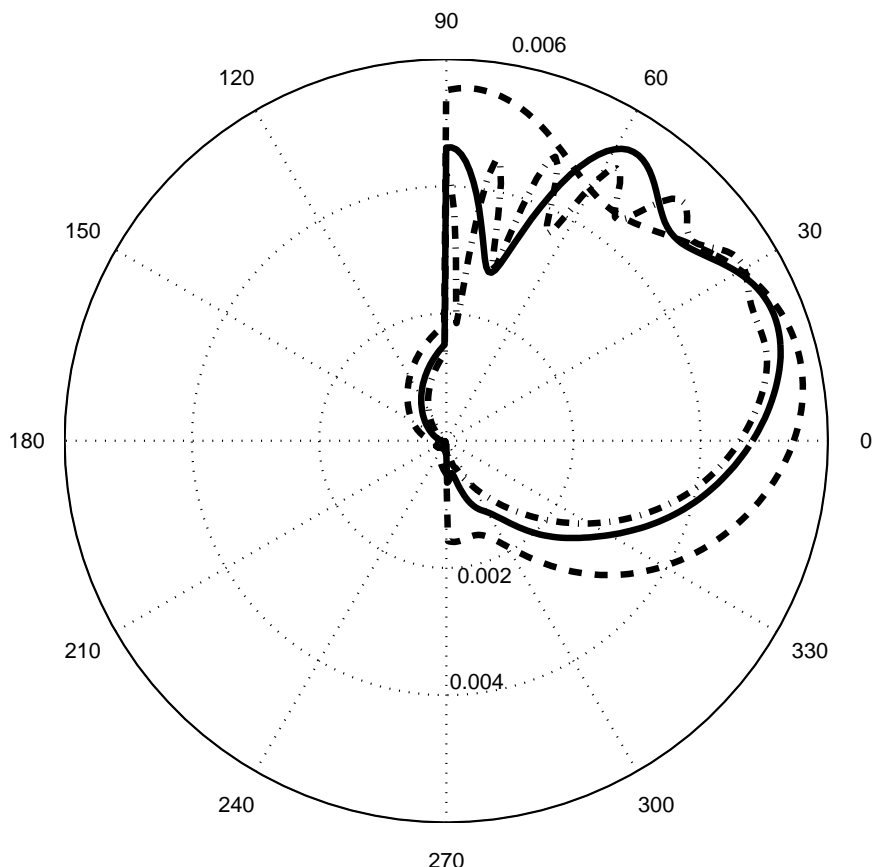

Fig. 9. Polar plot of the current density $\left|J_{z}\right|$ induced over the surface of an elliptic cylinder with $\xi_{1}=2$ for the perpendicular configuration when the source is an E-polarized plane wave incident at $\varphi_{0}=\pi / 6$ and $\zeta=1 / 3$. Results correspond to $c=1$ (dashed line), $c=\pi$ (solid line); $c=10$ (dashdot line). Observe the jump at $v=\pi / 2,3 \pi / 2$ due to $\zeta \neq 1$ at the interface between medium 1 and 2 .

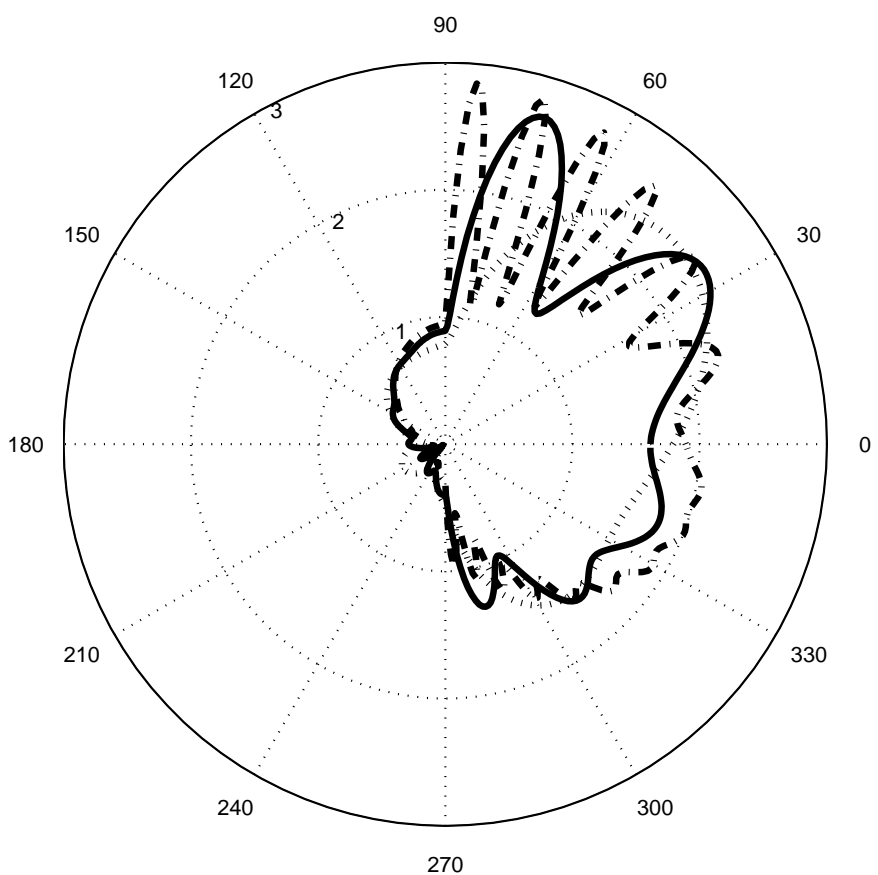

Fig. 10. Polar plot of the current density $\left|J_{v}\right|$ induced over the surface of an elliptic cylinder with $\xi_{1}=2$ for the perpendicular configuration when the source is an H-polarized plane wave incident at $\varphi_{0}=\pi / 6$ and $\zeta=1 / 3$. Results correspond to $c=1$ (dashed line), $c=\pi$ (solid line); $c=10$ (dashdot line). Observe the continuity at $v=\pi / 2,3 \pi / 2$ at the interface between medium 1 and 2 . 


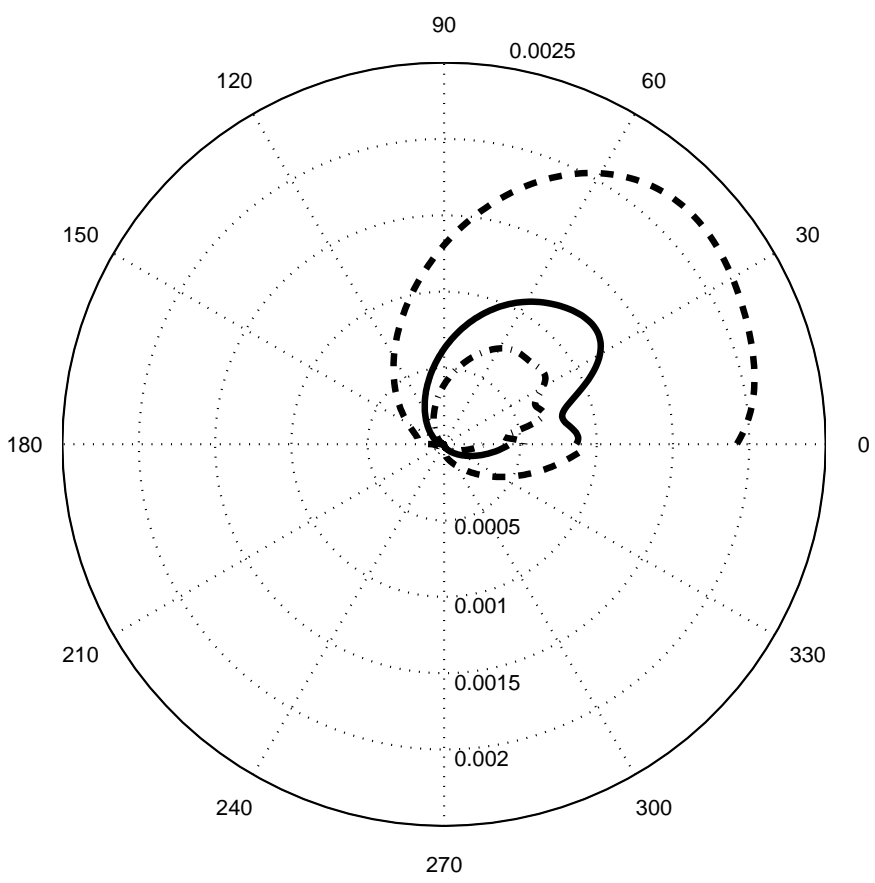

Fig. 11. Polar plot of the current density $\left|J_{z}\right|$ induced over the surface of a cylinder with $\xi_{1}=2$ for the parallel configuration when the source is an electric line located at $\left(\xi_{0}=6, v_{0}=\pi / 4\right)$ and $\zeta=1 / 2$. Results correspond to $c=1$ (dashed line), $c=\pi$ (solid line); $c=7$ (dash-dot line). Observe the jump at $v=0, \pi$ due to $\zeta \neq 1$ at the interface between medium 1 and 2 .

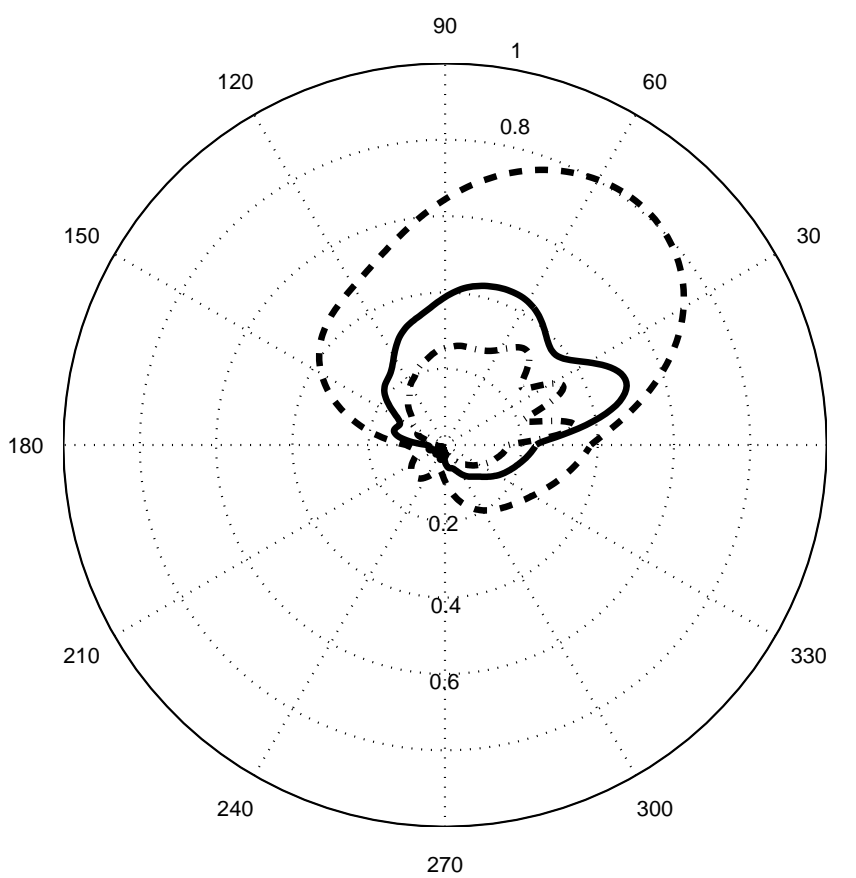

Fig. 12. Polar plot of the current density $\left|J_{v}\right|$ induced over the surface of an elliptic cylinder with $\xi_{1}=2$ for the parallel configuration when the source is a magnetic line located at $\left(\xi_{0}=6, v_{0}=\pi / 4\right)$ and $\zeta=1 / 2$. Results correspond to $c=1$ (dashed line), $c=\pi$ (solid line); $c=7$ (dash-dot line). Observe the continuity at $v=0, \pi$ at the interface between medium 1 and 2 .

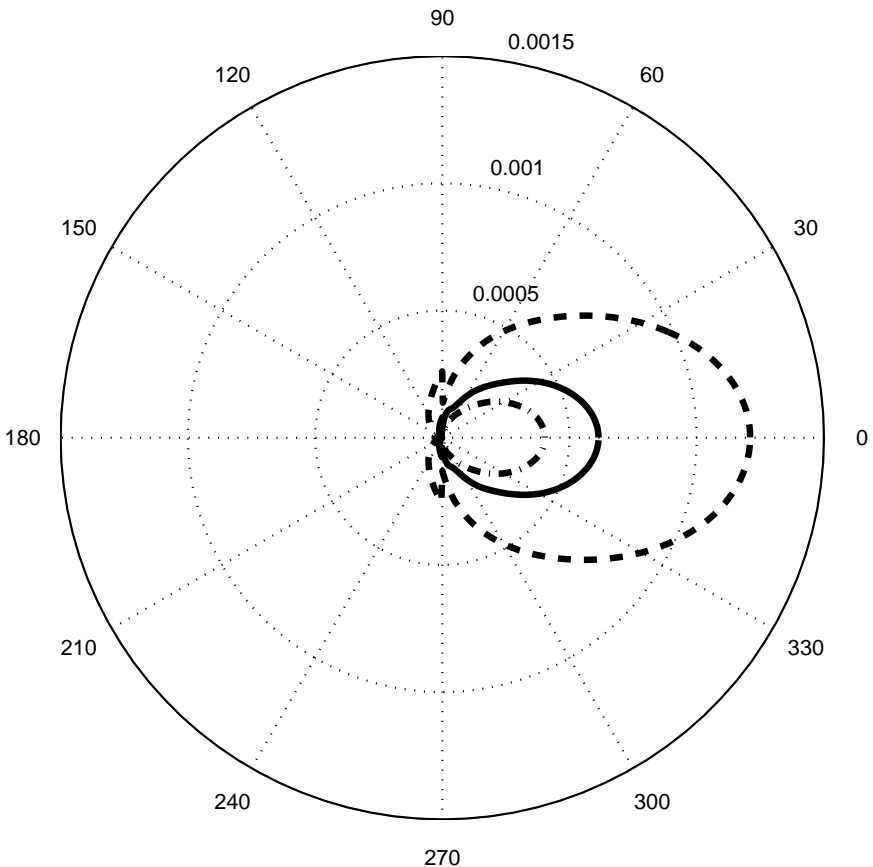

Fig. 13. Polar plot of the current density $\left|J_{z}\right|$ induced over the surface of a cylinder with $\xi_{1}=2$ for the perpendicular configuration when the source is an electric line located at $\left(\xi_{0}=6, v_{0}=0\right)$ and $\zeta=2$. Results correspond to $c=1$ (dashed line), $c=\pi$ (solid line); $c=7$ (dash-dot line). Observe the symmetry and the jump at $v=\pi / 2$ and $v=3 \pi / 2$ due to $\zeta \neq 1$ at the interface between medium 1 and 2 .

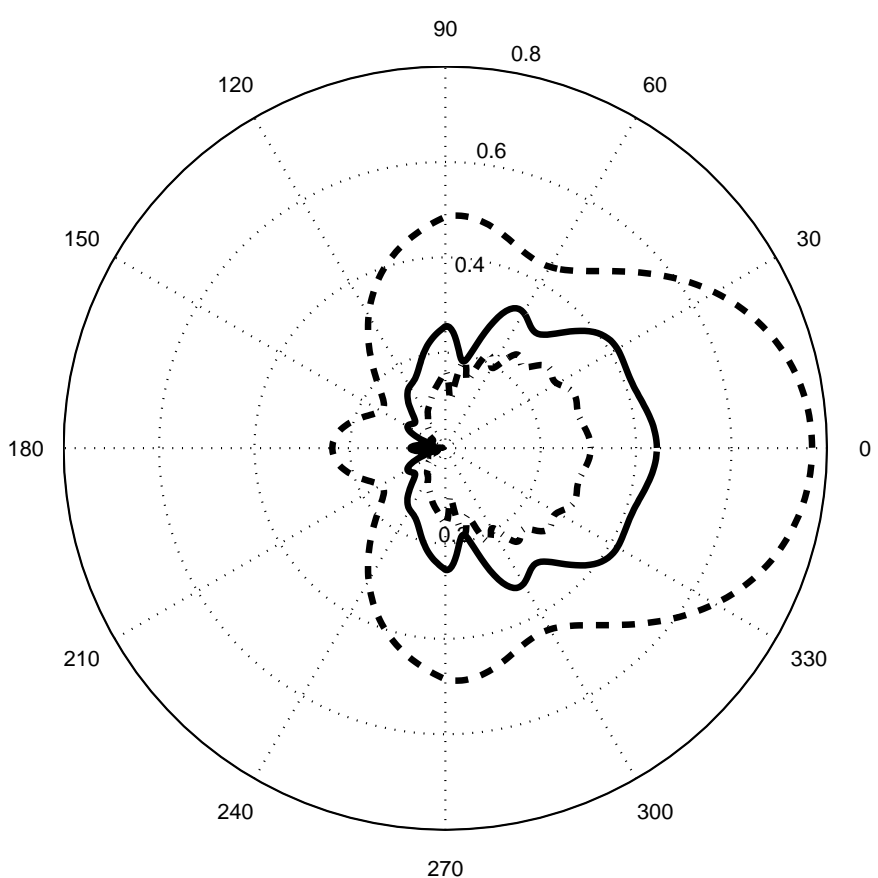

Fig. 14. Polar plot of the current density $\left|J_{v}\right|$ induced over the surface of an elliptic cylinder with $\xi_{1}=2$ for the perpendicular configuration when the source is a magnetic line located at $\left(\xi_{0}=6, v_{0}=0\right)$ and $\zeta=2$. Results correspond to $c=1$ (dashed line), $c=\pi$ (solid line); $c=7$ (dash-dot line). Observe the symmetry and the continuity at $v=\pi / 2$ and $v=3 \pi / 2$ at the interface between medium 1 and 2 . 
Fig. 8 shows the magnitude of the current density $J_{z}$ for the parallel configuration due to an $\mathrm{H}$-polarized plane wave incident at $\varphi_{0}=\pi / 6$ when $\zeta=1 / 3$. Contrary to Fig. 7, there is no jump in the value of $\left|J_{z}\right|$ across $v=0, \pi$, as can be easily seen from (39) and using the property $\left.\operatorname{So}_{m}(c, \eta)\right|_{v=0, \pi}=0$.

Fig. 9 shows the magnitude of the current density $J_{z}$ for the perpendicular configuration due to an E-polarized plane wave incident at $\varphi_{0}=\pi / 6$ when $\zeta=1 / 3$. There is a jump in the value of $\left|J_{z}\right|$ at $v=\pi / 2$ and $v=3 \pi / 2$. Referring to (46) and (29) with $h=2$, it is easy to verify (using properties (15)-(16)) that the quantities under the summation symbols are the same at $v=\pi / 2,3 \pi / 2$ and that the jump is related to the different intrinsic impedances of the two media. Fig. 10 shows the magnitude of the current density $J_{z}$ for the perpendicular configuration due to an $\mathrm{H}$-polarized plane wave incident at $\varphi_{0}=\pi / 6$ when $\zeta=1 / 3$. Contrary to the previous case, there is continuity in the value of $\left|J_{z}\right|$ at $v=\pi / 2,3 \pi / 2$.

The following four figures deal with the current induced over the surface of a PEC cylinder with size $\xi_{1}=2$ and due to line sources. Fig. 11 shows the magnitude of the current density $J_{z}$ for the parallel configuration due to an isotropic electric line located at $\left(\xi_{0}=6, v_{0}=\pi / 4\right)$ when $\zeta=1 / 2$. There is a jump in the value of $\left|J_{z}\right|$ at $v=0$ and $v=\pi$. In fact, from (58), in a neighborhood of $v=0$ or $v=\pi$ the contribution from the odd Mathieu functions is zero, since $\mathrm{So}_{m}(c, \pm 1)=0$, and the presence of $Z_{h}$ determines the dependence upon the medium that causes the jump, when $\zeta \neq 1$. Fig. 12 shows the magnitude of the current density $J_{z}$ for the parallel configuration due to an isotropic magnetic line located at $\left(\xi_{0}=6, v_{0}=\pi / 4\right)$ when $\zeta=1 / 2$. Contrary to the case of the electric line, this time there is no jump in the value of $\left|J_{z}\right|$ at $v=0$ and $v=\pi$. In fact, from (62), in a neighborhood of $v=0$ or $v=\pi$, the contribution from the odd Mathieu functions is zero, since $\operatorname{So}_{m}(c, \pm 1)=0$ and the dependence upon the medium disappears, regardless of the value of $\zeta$. Fig. 13 shows the magnitude of the current density $J_{z}$ for the perpendicular configuration due to an isotropic electric line located at $\left(\xi_{0}=6, v_{0}=0\right)$ when $\zeta=2$. The curves represented are symmetric because of the symmetry in the geometry as well as for the location of the line source. Similar to Fig. 11, there are two jumps: at $v=\pi / 2$ and at $v=3 \pi / 2$. In fact, using properties (15)-(16), the quantities inside the summation symbols in equations (67) and (68) are continuous around $v=\pi / 2,3 \pi / 2$. However, the coefficients outside the summation symbols are not the same and, hence, this causes a jump when $\zeta \neq 1$. Fig. 14 shows the magnitude of the current density $J_{z}$ for the perpendicular configuration due to an isotropic magnetic line located at $\left(\xi_{0}=6, v_{0}=0\right)$ when $\zeta=2$. The curves represented are symmetric because of the symmetry in the geometry as well as for the location of the line source. Contrary to Fig. 13 there no jumps: at $v=\pi / 2$ and at $v=3 \pi / 2$. In fact, using the properties (15)-(16), one can easily see that the quantities inside the summation symbols in (72) and (73) are the same around $v=\pi / 2,3 \pi / 2$ and that the coefficients that multiply the summation symbols are the same because of (11).

The computations for the line sources were carried out by separately applying Shanks transform to the real and imaginary parts of the terms of the series involved, similar to what is described in [20].

The last numerical examples consider the current induced over a strip by an H-polarized plane wave. Fig. 15 reproduces the results given in Fig. 4.18 of [15] for incidence at an angle $\varphi_{0}=\pi / 2$ and $\zeta=1$.

Figs. 16 and 17 examine the current induced over the surface of a strip that is illuminated by an $\mathrm{H}$-polarized plane wave at three different values of incidence: $\varphi_{0}=\pi / 6, \pi / 3, \pi / 2$ and for of $d / \lambda=0.45$ and $d / \lambda=1.27$. In all cases $\zeta=1$. These figures reproduce Fig. 4.16a-c and Fig. 4.16c-d of [15], respectively. The qualitative agreement is good, but there exist quantitative differences between Fig. 4.16-c of [15] and the top part of Fig. 16, as well as Fig. 4-16-a of [15] and Fig. 17. The results reported here have been verified and are correct. Therefore, the results presented herein are a correction to Fig. 4-16 of [15] as well as to its source reported in [21].

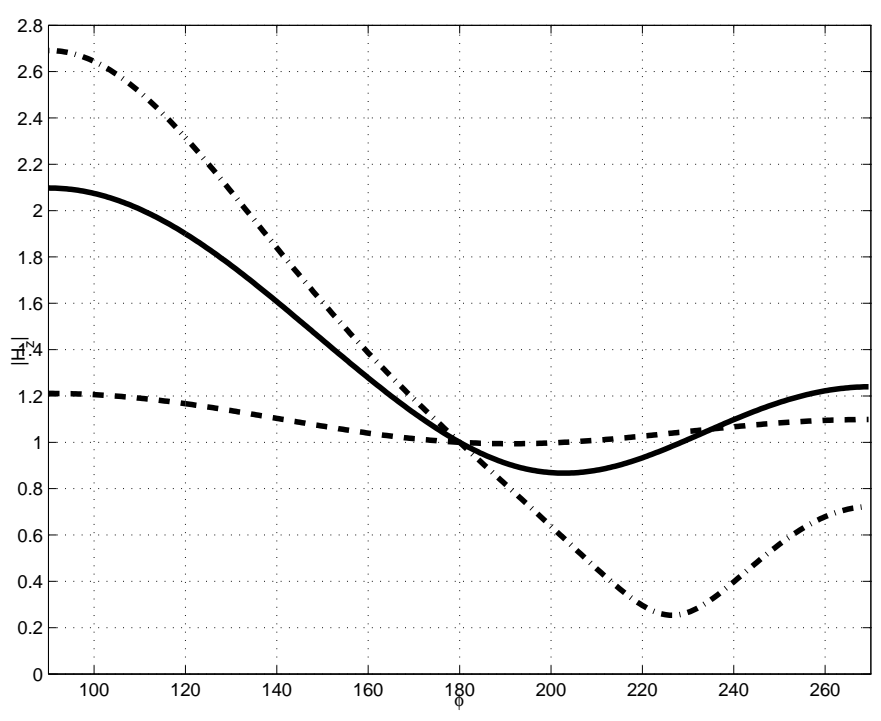

Fig. 15. Magnitude of the total magnetic field on a strip for an H-polarized plane wave incident at an angle $\varphi_{0}=\pi / 2$, with $\zeta=1$. Results correspond to $c=0.5$ (dashed line), $c=1$ (solid line); $c=2$ (dash-dot line).

\section{CONCLUSION}

The new canonical boundary-value problems solved herein enrich the catalog of exact solutions while providing a good testbed for the validation of frequency-domain computer codes. When the two isorefractive media are the same, i.e. $\zeta=1$, the solutions in this work become the known formulas for a metal elliptic cylinder immersed in a linear, homogeneous and isotropic medium [15].

\section{ACKNOWLEDGEMENTS}

The authors are thankful to the Reviewers for their comments that helped improve the quality of this paper.

\section{REFERENCES}

[1] P.L.E. Uslenghi, "Exact scattering by isorefractive bodies," IEEE Trans. Antennas Propagat., vol. 45, no. 9, pp. 1382-1385, Sept. 1997. 
[2] L. Knockaert, F. Olyslager, and D. DeZutter, "The diaphanous wedge," IEEE Trans. Antennas Propagat., vol. 45, no. 9, pp. 1374-1381, Sept. 1997.

[3] P.L.E. Uslenghi, "Special solutions for isorefractive wedge," in Proc. Intl. Conf. on Electromagnetics in Advanced Applications (ICEAA'01), Torino, Italy, Sept. 2001, pp. 421-423.

[4] V.G. Daniele and P.L.E. Uslenghi, "Closed-form solution for a line source at the edge of an isorefractive wedge," IEEE Trans. Antennas Propagat., vol. 47, no. 4, pp. 764-765, April 1999.

[5] R.W. Scharstein and A.M.J. Davis, "Time-domain three-dimensional diffraction by the isorefractive wedge," IEEE Trans. Antennas Propagat., vol. 46, no. 8, pp. 1148-1158, Aug. 1998.

[6] P.L.E. Uslenghi and R.E. Zich, "Radiation and scattering from isorefractive bodies of revolution," IEEE Trans. Antennas Propagat., vol. 46 no. 11, pp. 1606-1611, Nov. 1998.

[7] S. Roy and P.L.E. Uslenghi, "Exact scattering for axial incidence on an isorefractive paraboloid," IEEE Trans. Antennas Propagat., vol. 45, no. 10, p. 1563 , Oct. 1997

[8] P.L.E. Uslenghi, "Exact penetration, radiation and scattering for a slotted semielliptical channel filled with isorefractive material," IEEE Trans. Antennas Propagat., accepted for publication.

[9] D. Erricolo and P.L.E. Uslenghi, "Penetration, radiation and scattering for a cavity-backed gap in a corner," IEEE Trans. Electromagn. Compat., 2003, submitted.

[10] C. Berardi, D. Erricolo, and P.L.E. Uslenghi, "Exact dipole radiation for an oblate spheroidal cavity filled with isorefractive material and aperture-coupled to a half space," IEEE Trans. Antennas Propagat., 2003, submitted.

[11] D. Erricolo, M.D. Lockard, C.M. Butler, and P.L.E. Uslenghi, "Numerical analysis of penetration, radiation and scattering for a slotted semielliptical channel filled with isorefractive material," IEEE Trans. Antennas Propagat., 2003, submitted.

[12] D. Erricolo, M.D. Lockard, C.M. Butler, and P.L.E. Uslenghi, "Currents on conducting surfaces of a semielliptical-channel-backed slotted screen in an isorefractive environment," IEEE Trans. Antennas Propagat., 2003, submitted.

[13] D. Erricolo, M.D. Lockard, C.M. Butler, and P.L.E. Uslenghi, "Comparison among currents on surfaces inside and near a semielliptical channel filled with isorefractive material that backs a slotted plane: currents computed by analytical formulas and by integral equation methods," in Proc. Intl. Conf. on Electromagnetics in Advanced Applications (ICEAA'03), Torino, Italy, Sept 2003.

[14] J. A. Stratton, Electromagnetic Theory, McGraw-Hill, New York, 1941.

[15] J.J. Bowman, T.B.A. Senior, and P.L.E. Uslenghi, Electromagnetic and Acoustic Scattering by Simple Shapes, Hemisphere Publishing Corporation, New York, 1987.

[16] S. Zhang and J. M. Jin, Computation of Special Functions, Wiley, New York, 1996.

[17] S. Goldstein, "Mathieu functions," Camb. Phil. Soc. Trans., vol. 23, pp. 303-336, 1927.

[18] E. L. Ince, "Tables of elliptic cylinder functions," Roy. Soc. Edin. Proc., vol. 52, pp. 355-423, 1932

[19] M. Abramovitz and I. A. Stegun, Handbook of Mathematical Functions, Dover Publications, Inc, New York, 1970.

[20] D. Erricolo, "Acceleration of the convergence of series containing Mathieu functions using Shanks transformation," IEEE Antennas and Wireless Propagation Letters, vol. 2, pp. 58-61, 2003.

[21] H. P. Hsu, "Aperture fields in the diffraction by a slit," J. Appl. Phys., vol. 31, pp. 1742-1746, 1959.

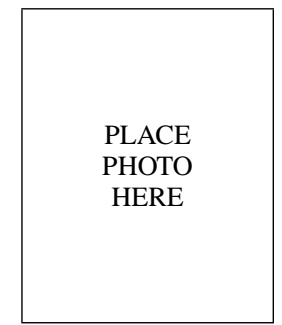

Danilo Erricolo (S'97 - M'99) received the Laurea degree of Doctor in Electronics Engineering (summa cum laude) from the Politecnico di Milano, Italy, in 1993, and the Ph.D. degree in Electrical Engineering and Computer Science from the University of Illinois at Chicago in 1998. He is a research scientist and a lecturer in the College of Engineering of the University of Illinois at Chicago where he also is the Associate Director of the Electromagnetics Laboratory. He is the Senior Associate Editor for the IEEE Antennas and Wireless Propagation Letters. His research interests are in computational electromagnetics, wireless communications, electromagnetic scattering, and electromagnetic compatibility. $\mathrm{He}$ has authored or co-authored more than 50 publications in international journals and conferences. Dr. Erricolo was awarded twice both the Andrew Foundation Fellowship and the Beltrami Foundation Fellowship. In 2002 he was elected full member of the United States National Committee of the International Union of Radio Science (USNC-URSI) Commission B.

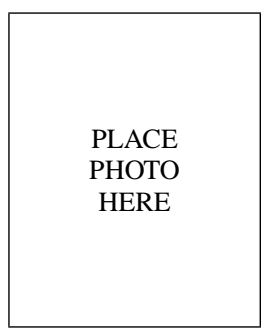

Piergiorgio L. E. Uslenghi (SM '70 - F '90) For biography and photo, see this Transactions, June 2004. 

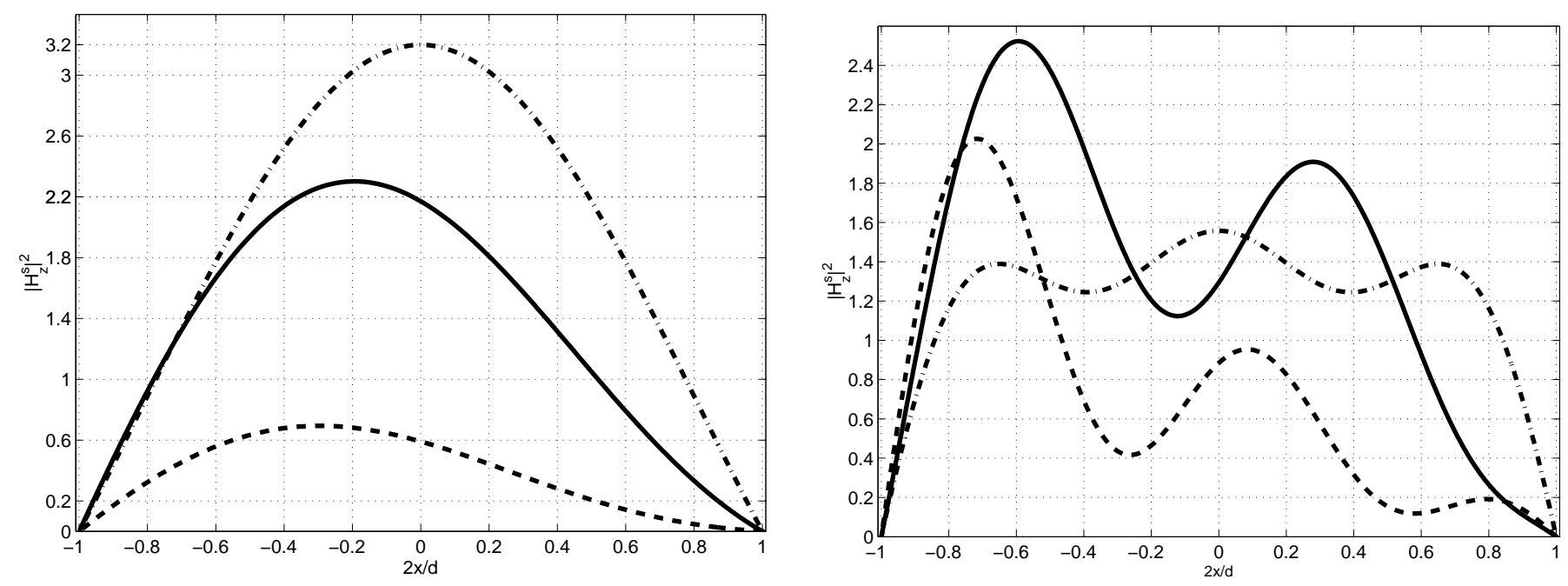

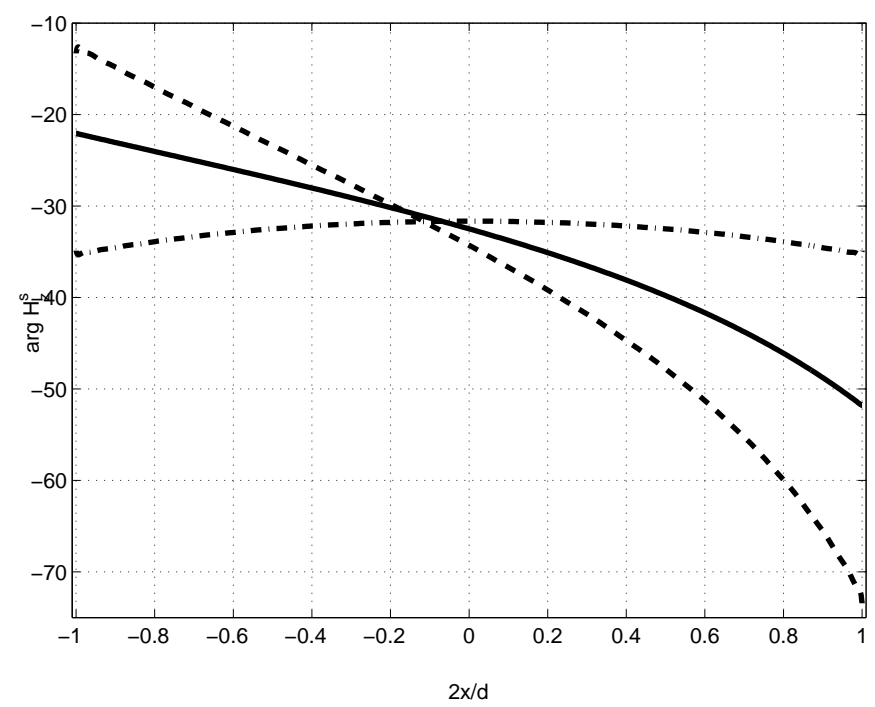

Fig. 16. Scattered magnetic field $H_{1 z}(x)$ due to an H-polarized plane wave incident at $\varphi_{0}=\pi / 6$, dashed-line, $\varphi_{0}=\pi / 3$, solid-line, and $\varphi_{0}=\pi / 2$ dash-dot line. The top part of this figure shows $\left|H_{1 z}(x)\right|^{2}$ and the lower part the shows the argument of $\left|H_{1 z}(x)\right|$ when $d / \lambda=0.45$.

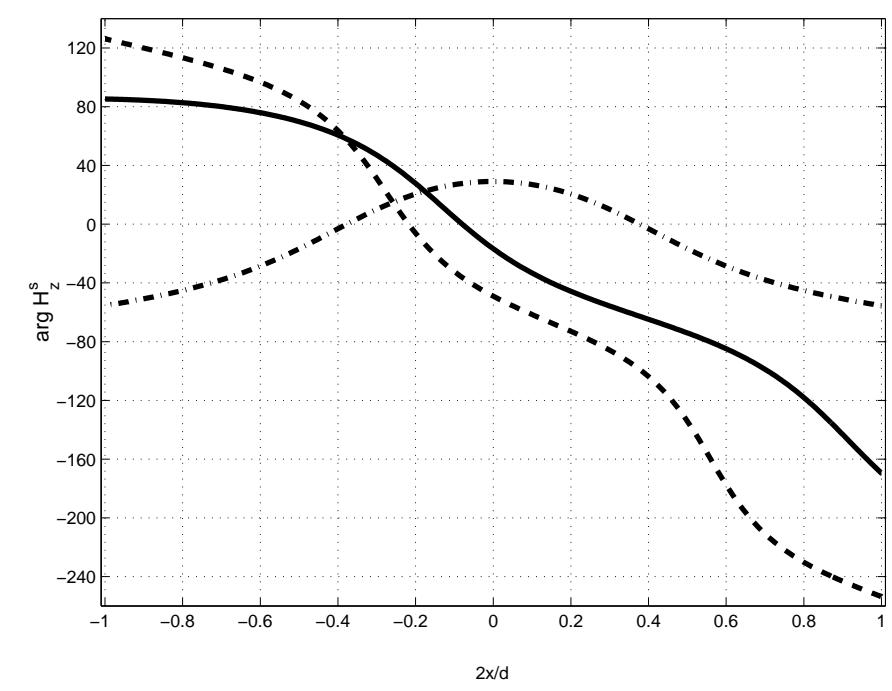

Fig. 17. Scattered magnetic field $H_{1 z}(x)$ due to an H-polarized plane wave incident at $\varphi_{0}=\pi / 6$, dashed-line, $\varphi_{0}=\pi / 3$, solid-line, and $\varphi_{0}=\pi / 2$ dash-dot line. The top part of this figure shows $\left|H_{1 z}(x)\right|^{2}$ and the lower part the shows the argument of $\left|H_{1 z}(x)\right|$ when $d / \lambda=1.27$. 